\title{
Circumferential MFL IN-LINE INSPECTION FOR CRACKS IN PIPELINES
}

\section{FINAL REPORT}

\author{
J. B. Nestleroth \\ Battelle \\ 505 King Avenue \\ Columbus, Ohio 43201
}

June 2003

Grant/Cooperative Agreement No. DE-FC26-01NT41159

Prepared for the

Department of Energy

National Engineering Technology Laboratory 


\section{Disclaimer}

This report was prepared as an account of work sponsored by an agency of the United States Government. Neither the United States Government nor any agency thereof, nor any of their employees, makes any warranty, express or implied, or assumes any legal liability or responsibility for the accuracy, completeness, or usefulness of any information, apparatus, product, or process disclosed, or represents that its use would not infringe privately owned rights. Reference herein to any specific commercial product, process, or service by trade name, trade mark, manufacturer, or otherwise does not necessarily constitute or imply its endorsement, recommendation, or favoring by the United States Government or any agency thereof. The views and opinions of authors expressed herein do not necessarily state or reflect those of the United States Government or any agency thereof. 


\section{Executive Summary}

The first in-line tools used magnetic flux leakage (MFL) to detect metal-loss corrosion, and MFL tools are still the most common inspection tools used by natural gas and liquid pipeline companies. However, conventional MFL tools have significant limitations. Most notably, the traditional implementation of MFL tools cannot reliably detect or size axially aligned cracks.

The inability of current tools to reliably detect long narrow defects is not so much a limit of MFL technology as a limit with the standard implementation. Circumferential MFL is a new implementation that has potential to detect and quantify axially oriented defects such as cracks, seam weld defects, mechanical damage, and groove corrosion. This implementation works by orienting the magnetic field around the pipe rather that along the axis. By orienting the magnetic field around the pipe (the circumferential direction), the axial defects that were magnetically transparent disrupt more of the magnetic field and can be more easily detected. Recent advances in unrelated industries have made a new implementation of this technology feasible.

To further advance circumferential MFL technology, inspection and analysis methodologies that improve detection capability and sizing accuracy for cracks were examined. Initial implementations of circumferential MFL have found that flux leakage from cracks is small, and the signals from cracks at the interior of the pipe are difficult to detect. The objective of this project was to improve detection of cracks by changing the implementation along with using data from overlapping and complementary inspection techniques. Two technology enhancements were investigated:

- Combining high- and low-magnetization technology for stress detection

- Combining axial and circumferential MFL methods.

Full-scale experiments were used to test the technology enhancements. The experiments used existing defect sets, previously developed equipment, and new components. Where appropriate, this project used equipment that is part of the Pipeline Safety and Reliability Center (PSRC). The PSRC supplied both natural SCC samples and pipe with artificial flaws. A new circumferential MFL tool including sensors was built that uses a PSRC-supplied MFL data recording module. To conduct the tests under pressure, a special apparatus was designed and configured that used PSRC Flow Loop compressors to provide the high-pressure gas.

Magnetization is a significant inspection variable, since it is much more difficult to saturate the pipe material in the circumferential direction. The strength of the magnetic field is greatest near the magnetizer poles and smallest at the center. This is caused by the poor magnetic properties of the pipe material in the circumferential direction and the circular geometry providing alternative flux paths. This non-uniform field makes defect sizing more difficult. A defect near one magnet pole gives a different signal than the same defect equidistant from magnets. Compensation methods are used to adjust the amplitudes to an average magnetization level. Velocity effects for circumferential MFL are detectable at lower speeds and are more significant than for axial MFL. Velocity induced signal changes are detected at speeds of $1.0 \mathrm{~m} / \mathrm{s}(2.2 \mathrm{mph})$. If the defect is midway between the poles, the amplitude is simply reduced. However, if the defect is closer to one pole, the flux leakage is also distorted. At inspection speeds of $1.0 \mathrm{~m} / \mathrm{s}$ $(2.2 \mathrm{mph})$, the magnetizing field starts to concentrate at the inner surface of the pipe, making detection of OD defects more difficult. 
The technology developed and tested on this project provides only limited improvements to inspection capability. Although a method combining high- and low-magnetization technology showed promise, its commercial development was not successful for two reasons. First, the stress diminishes the crack signal, while the opening of the crack increases the signal. The stress-induced changes in flux leakage around cracks were small and any critical information on the severity of cracks and crack-like defects is difficult to distinguish from changes caused by the crack opening and other inspection variables. Second, it is difficult to magnetize pipe material in the circumferential direction. A relatively low, non-uniform magnetization level produced by the circumferential magnetizer makes detection of changes due to stress extremely difficult. This project also examined combining axial and circumferential MFL to improve crack detection and distinguish cracks for axially oriented volumetric defects. While successful results are presented in this report, circumferential MFL can only detect larger cracks. Even with the field aligned properly, circumferential MFL technology has difficulty detecting all cracks on the outside surface that have the potential to grow to failure within a normal inspection interval of 5 to 10 years.

Circumferential MFL can be used to detect many corrosion, mechanical damage, and crack defects. However, the detection capabilities and sizing accuracies may not be sufficient for all pipeline threats. Circumferential MFL will be useful in identifying locations for detailed testing. Inspection tools that use more sophisticated technologies for detecting and sizing defects may have better performance capabilities, but will likely be expensive to operate. While circumferential MFL and the performance enhancements developed on this project may have limitations, this inspection method will be part of the inspection process for many decades. 


\section{Table of Contents}

List of Acronyms, Initialisms, and Abbreviations ............................................................ ix

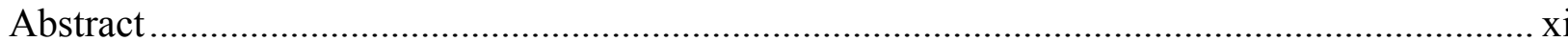

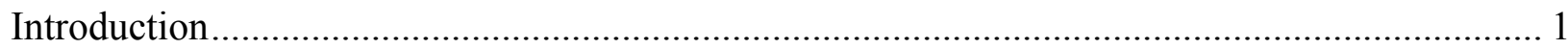

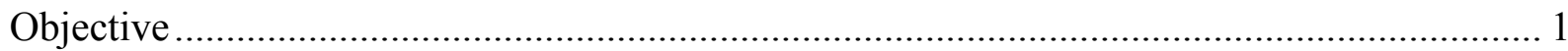

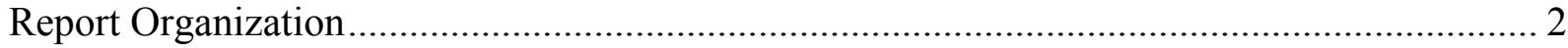

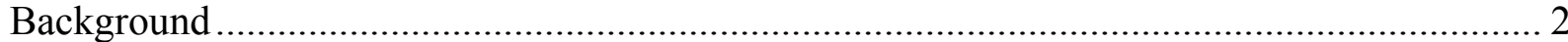

Cracks and Their Inspection-Related Characteristics .................................................. 2

Important Features of Crack-Like Defects ............................................................... 2

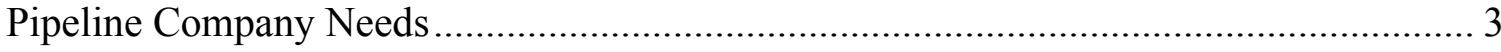

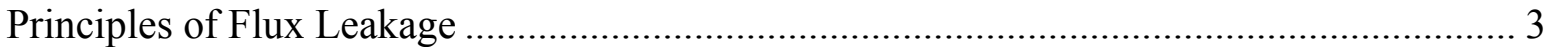

Flux Leakage Caused by Metal Loss ............................................................................. 3

Flux Leakage Caused by Stress and Magnetic Property Changes................................. 3

Flux Leakage Caused by Thin Crack-like Defects ........................................................... 4

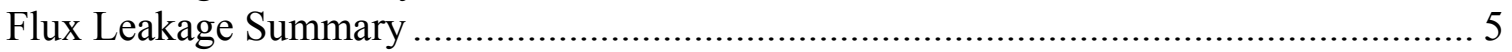

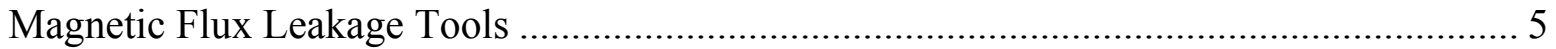

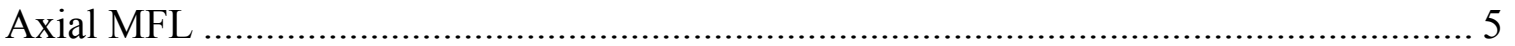

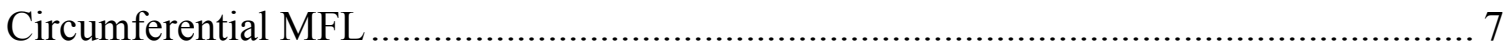

Prior Circumferential MFL Developments .............................................................. 9

Other Developments Aimed at Detection of Axial Cracks........................................ 10

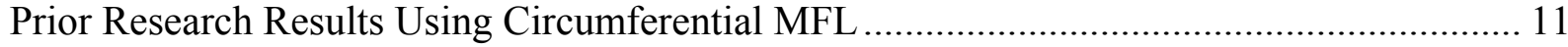

Stress Corrosion Cracks and Weld Corrosion .................................................................. 11

SCC — Unstressed Conditions ....................................................................... 11

SCC — Stressed Conditions ........................................................................... 12

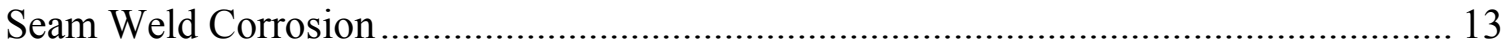

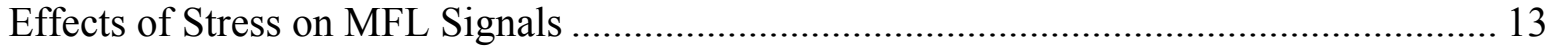

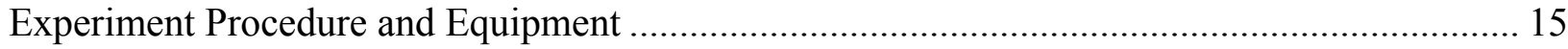

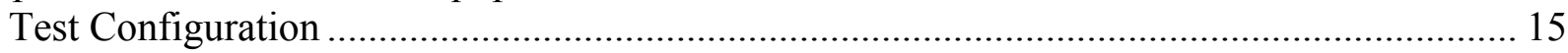

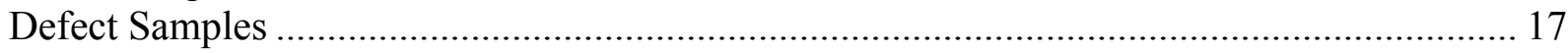

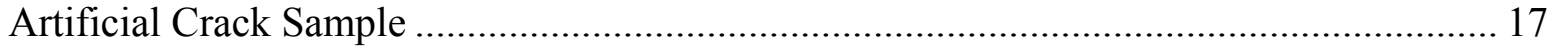

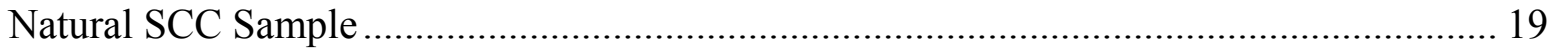

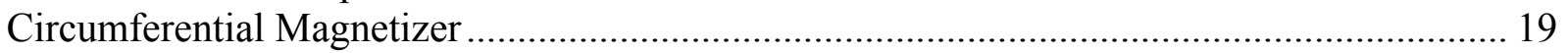

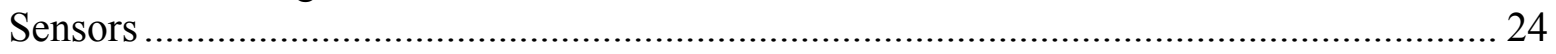

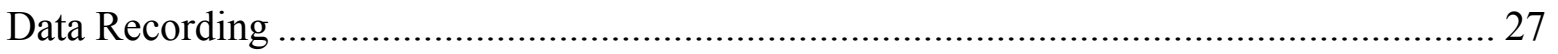

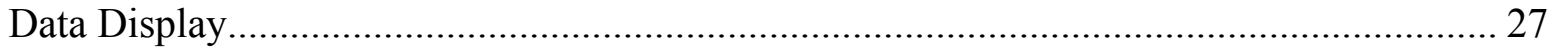

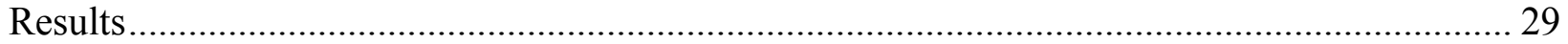

High and Low Magnetization Technology ..................................................................... 29

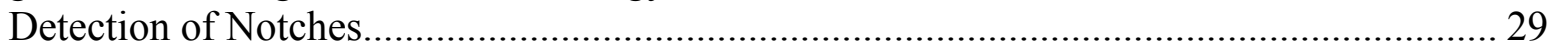

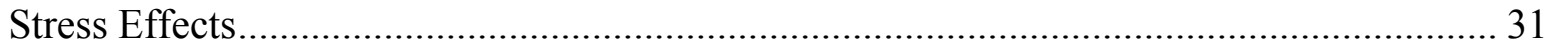

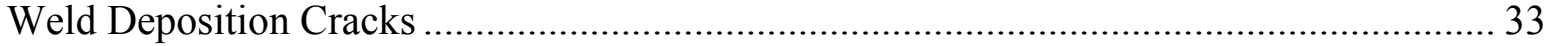

Natural Stress Corrosion Cracks............................................................................... 34

Combining Circumferential and Axial MFL .................................................................. 35

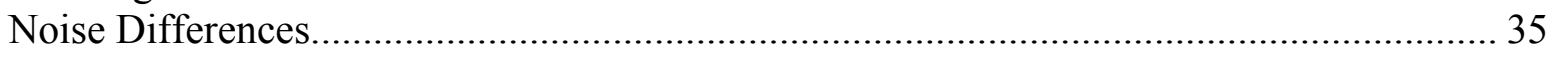

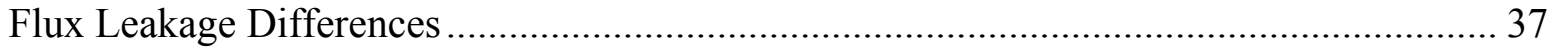

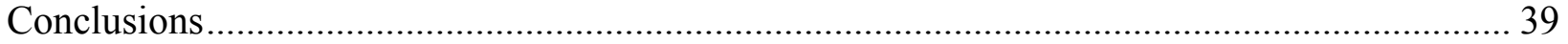

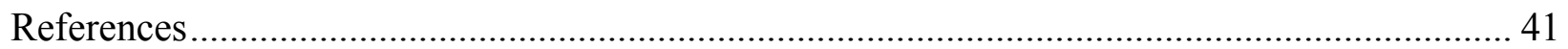




\section{List of Tables}

Page

Table 1. Crack Depths................................................................................................ 33

\section{List of Figures}

Figure 1. The effect of stresses on the magnetization curves which govern magnetism and flux leakage.

Figure 2. A simple dipole model of a crack in a magnetic field.

Figure 3. Typical axial MFL tool design.

Figure 4. Schematic and typical execution of circumferential MFL tool design......

Figure 5. In-situ magnetic measurements demonstrating difficulty in saturating the pipe material in the circumferential direction.

Figure 6. The strength of the magnetic field is greatest near the magnetizer poles and smallest at the center.

Figure 7. Results of a magnetic particle inspection of the outer pipe surface.

Figure 8. Circumferential MFL results.....

Figure 9. Circumferential MFL signals under pressure.

Figure 10. Circumferential MFL signals from selective seam corrosion................................. 13

Figure 11. The effect of compressive residual stresses................................................ 14

Figure 12. Apparatus to acquire data at multiple pressures and multiple magnetization levels. 16

Figure 13. Example of EDM notch and weld deposition crack defects ............................. 18

Figure 14. Stress at and near the notch tip. ............................................................. 19

Figure 15. Electromagnet circumferential magnetizer............................................ 20

Figure 16. The circumferential component of the magnetic field around the circumference of the pipe for the electromagnet test bed vehicle circumferential magnetizer..... 21

Figure 17. The radial component of the magnetic field around the circumference of the pipe for the electromagnet test bed vehicle circumferential magnetizer.

Figure 18. In-situ magnetic measurements demonstrating difficulty in saturating the pipe material in the circumferential direction.

Figure 19. Quarter magnetic finite element model electromagnet circumferential magnetizer. 23

Figure 20. Field level in the LTR pipe segment as a function of current level. ................... 24

Figure 21. A sensor head with replaceable sensor circuit. ........................................ 25

Figure 22. An early sensor system with four sensors per sensor head............................... 25

Figure 23. Sensor spacing determination based on crack signals acquired in the laboratory at 0.050 -inch circumferential intervals.

Figure 24. Dense sensor spacing for crack detection experiments with 16 sensors per one inch wide sensors head.

Figure 25. The data recording system (left) is mounted in a shock and vibration damping cage (right).

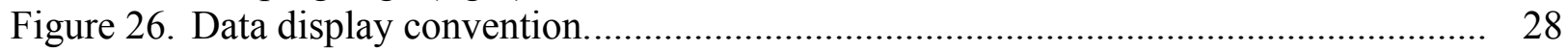

Figure 27. Flux leakage for the full pipe sample with EDM notches. ............................... 30

Figure 28. Flux leakage from three EDM notches demonstrating the detectability of these defects.

Figure 29. Flux leakage from a weld zone with EDM notches.

Figure 30. Flux leakage signals from a fifty percent deep EDM notch at pressures ranging from atmospheric to approaching yield. 
Figure 31. Flux leakage from weld deposition cracks.

Figure 32. A flux leakage image, magnetic particle enhanced photograph of a stress corrosion crack colony and a photograph indicating corrosion pits.

Figure 33. Close-up of a flux leakage image and magnetic particle enhanced photograph of a stress corrosion crack colony.

Figure 34. Single channel axial and circumferential flux leakage data showing both the noise differences and the repeatability of multiple pulls. 36

Figure 35. Axial and circumferential flux leakage image showing both the noise differences. 37

Figure 36. Circumferential and axial flux leakage image of stress corrosion crack colonies and corrosion pits 


\title{
LIST OF ACRONYMS, INITIALISMS, AND ABBREVIATIONS
}

\author{
EDM electro discharge machining \\ EMAT electromagnetic acoustic transducer \\ ERW electric-resistance welded \\ MFL magnetic flux leakage \\ PRCI Pipeline Research Council International, Arlington, VA \\ PSRC Pipeline Safety and Reliability Center, Battelle, Columbus, $\mathrm{OH}$ \\ SCC stress-corrosion cracking \\ SwRI Southwest Research Institute, San Antonio, TX
}




\section{ABSTRACT}

Circumferential MFL is a new implementation of a widely used technology that has potential to provide improved detection and quantification of axially oriented defects such as cracks, seam weld defects, mechanical damage, and groove corrosion. This implementation works by orienting the magnetic field around the pipe rather that along the axis. By orienting the magnetic field around the pipe (the circumferential direction), the axial defects that were magnetically transparent can disrupt more of the magnetic field and can be more easily detected.

Initial implementations of circumferential MFL have found that flux leakage from cracks at the interior of the pipe is small, and the signals from cracks are difficult to detect. The objective of this project is to improve detection of cracks by changing the implementation along with using data from overlapping and complementary inspection techniques. Two technology enhancements were investigated:

- Combining high- and low-magnetization technology for stress detection

- Combining axial and circumferential MFL methods.

Although a method combining high- and low-magnetization technology showed promise for characterizing gouges cause by third party excavation equipment, its commercial development was not successful for two reasons. First, the stress diminishes the crack signal, while the opening of the crack increases the signal. The stress-induced changes in flux leakage around cracks were small and any critical information on the severity of cracks and crack-like defects is difficult to distinguish from changes caused by the crack opening and other inspection variables. Second, it is difficult to magnetize pipe material in the circumferential direction. A relatively low, non-uniform magnetization level produced by the circumferential magnetizer makes detection of changes due to stress extremely difficult. This project also examined combining axial and circumferential MFL to improve crack detection and distinguish cracks for axially oriented volumetric defects. While successful results are presented in this report, circumferential MFL can only detect larger cracks. Even with the field aligned properly, circumferential MFL technology has difficulty detecting cracks on the outside surface that have the potential to grow to failure.

Circumferential MFL can be used to detect many corrosion, mechanical damage, and crack defects. However, the detection capabilities and sizing accuracies may not be sufficient for all pipeline threats. Inspection tools that use more sophisticated technologies for detecting and sizing defects may have better performance capabilities, but will likely be expensive to operate. Circumferential MFL will be useful in identifying locations for detailed testing. While performance enhancements may be limited, circumferential MFL inspections will be part of the inspection process for many decades. 


\section{INTRODUCTION}

In-line inspection tools have been available for over 30 years. The first in-line tools used magnetic flux leakage (MFL) to detect metal-loss corrosion, and MFL tools are still the most common inspection tools used by natural gas and liquid pipeline companies. However, MFL tools cannot find nor were they designed to find all pipeline defects. Most notably, the traditional implementation of MFL tools cannot reliably detect or size axially aligned cracks.

The inability of current tools to reliably detect long narrow defects is not so much a limit of MFL technology as a limit with the standard implementation. Circumferential MFL is a new implementation that has potential to detect and quantify axially oriented defects such as cracks, seam weld defects, mechanical damage, and groove corrosion. This implementation works by orienting the magnetic field around the pipe rather that along the axis. By orienting the magnetic field around the pipe (the circumferential direction), the axial defects that were magnetically transparent disrupt more of the magnetic field and can be more easily detected. Recent advances in unrelated industries have made a new implementation of this proven technology feasible.

\section{OBJECTIVE}

Stress corrosion cracks (SCC) on pipelines form and grow on the external surface of the pipe. These cracks are tight, irregular, and can have branching with iron oxides between the fracture surfaces [1]. Flux leakage from cracks is small, and the signals from cracks at the interior surface are difficult to detect. The objective of this project is to improve detection of cracks using flux leakage methods along with data from overlapping and complementary inspection techniques. Two technology enhancements could provide the needed information:

- Combining high- and low-magnetization technology for stress pattern detection

- Combining axial and circumferential MFL methods.

In this project, inspection and analysis methodologies that improve detection and sizing accuracy for cracks using both of these enhancements were examined. Combining high- and lowmagnetization technology, as is being done for mechanical damage, will allow detection of stress patterns around defects; these stress patterns may contain critical information on the severity of cracks and crack-like defects. Combining axial and circumferential MFL in a novel fashion may enhance detection by identifying sources of signal considered noise by a single technique and by allowing the effects of magnetic noise to be reduced during signal processing.

MFL systems have many attributes. The systems can be designed to remain functional in an abusive pipeline environment for long distances at product flow speeds. The source of inspection energy (permanent magnets) requires no power during an inspection and the sensors and data recorders require reasonably low power to operate. The magnetic flux naturally enters the pipe and distributes evenly to produce a full volumetric inspection. While MFL has its attributes, it does not address all inspection needs. Developing methods and implementations that improve and augment this inspection technology would provide more reliable delivery of energy products and improved safety of pipeline systems. 


\section{REPORT ORGANIZATION}

This report reviews flux leakage principles as they apply to pipeline inspection for cracks and volumetric defects. This project also builds on flux leakage research, which is then reviewed. This is followed by a description of the experiment procedure and equipment. Finally, the results of high- and low-magnetization technology and combining circumferential and axial MFL are given.

\section{BACKGROUND}

\section{Cracks and Their Inspection-Related Characteristics}

Numerous cracks and crack-like defects can occur on pipelines. When discussing crack-like defects that can cause failures on pipelines, two categories dominate: seam weld defects and stress-corrosion cracking (SCC). In the seam weld category, most of the problems occur in electric resistance welded (ERW) pipe or flash welded pipe.

The most common defects in ERW and flash welded pipe are stitched welds (welds that are intermittently fused), hook cracks (cracks that are open to the inside or outside surface and which are oriented along planes of weakness in the pipe steel), and seam corrosion (external cracks that result from preferential corrosion of the bond line). Two forms of SCC are common: high-pH and near neutral $\mathrm{pH}$ (sometimes referred to as low-pH). These types of SCC differ in their morphology and driving mechanisms, but both can exist individually or in groups [1].

\section{Important Features of Crack-Like Defects}

From an inspection perspective, several features of crack-like defects are important. First, the separation between crack faces is critical. Cracks that are open (i.e., separated crack faces) are much easier to detect than cracks that are closed (tight). SCC and stitched ERW welds are typically very tight, making their detection and sizing difficult. Cracks that are open on the outside surface are less difficult to detect and size but are still problematic. Cracks that are open to the inside surface are easiest to detect and size.

A second feature of crack-like defects that is important during inspections is the proximity of adjacent defects and pipeline features. SCC often occurs in colonies, confusing the inspection signals and making data interpretation difficult. Seam-weld defects, by definition, occur near seam welds, which often introduce a geometric discontinuity. This discontinuity also makes signal interpretation difficult.

The efforts in this program concentrated on improved detection of tight cracks, cracks on the outside surface, and colonies of SCC. 


\section{Pipeline Company Needs}

Pipeline operators need methods that help ensure the integrity and safety of pipeline systems. Currently available inspection tools often cannot practically or reliably both detect and size many defects, such as axially aligned cracks. So, pipeline operators are left with no reliable and costeffective manner of detecting crack-like defects using in-line inspection tools.

One alternative to in-line inspection for axially oriented defects does exist: hydrotesting. Hydrotesting has been used successfully to uncover cracks in some areas, but it is expensive and time-consuming and it introduces potential environmental problems associated with disposal of the water. Consequently, there is a strong need for a successful in-line inspection alternative that can reliably detect cracks.

\section{Principles of Flux Leakage}

The flux leakage inspection method relies on a strong magnetic field interacting with a defect and sensors to detect the magnetic flux that leaves the pipe material [2]. In this report, the interaction of magnetic field with metal loss defects, stress and magnetic property changes, and thin crack-like defects will be discussed. The specific models used to explain signals caused each of these three conditions are summarized below.

\section{Flux Leakage Caused by Metal Loss}

MFL tools used for corrosion inspection use high magnetic fields to saturate the pipe material. At metal loss defects, the remaining material attempts to carry an increased amount of magnetic flux. This alone would be sufficient reason for flux to leak from pipe. But a second phenomenon causes even more flux to leak. In magnetically saturated materials, additional flux causes the flux carrying capability (the permeability) to decrease [3]. This double effect of increased flux and decreased capacity make for strong flux leakage signals.

\section{Flux Leakage Caused by Stress and Magnetic Property Changes}

Stress and material variation change the flux carrying capability of magnetic materials [4-6]. A local decrease in flux carrying capability will cause a signal similar to metal loss signals. A local increase in flux carrying capability will cause a decrease in signal amplitude. However, if the change in flux carrying capability is larger than the length of the magnetizer, as in the case of axial stress, the amount of flux in the pipe will be altered. For tensile stresses, the overall flux levels in the pipe will increase, while the flux will decrease for compressive stresses.

Figure 1 shows the effect of stresses and magnetic property changes on the magnetization curves which govern magnetism and flux leakage. These curves show the flux density variations for a given input field level become small for magnetic field levels greater than 120 Oersted. Most inspection tools are designed to operate above these field levels due to magnetic material variation. However, to detect stress changes, a magnetizer must operate at lower field levels. But field levels below the knee of the magnetization curve, typically about 40 Oersted, produce 
June 2003

results that are difficult to interpret. Most successful attempts to study stress effects have used fields around 50 to 70 Oersted $[7,8]$.

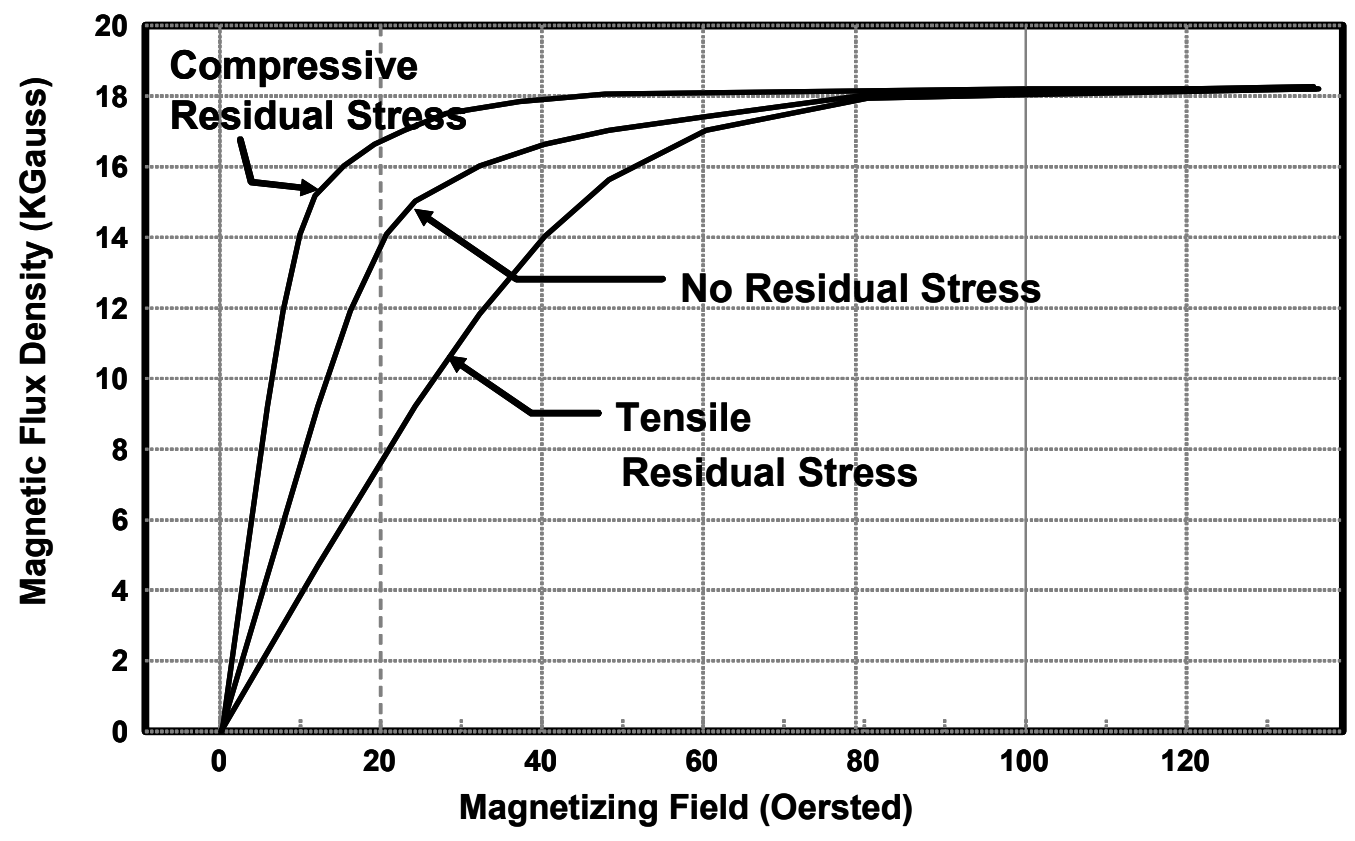

Figure 1. The effect of stresses on the magnetization curves which govern magnetism and flux leakage.

\section{Flux Leakage Caused by Thin Crack-like Defects}

A model of a crack in a magnetic field is illustrated in Figure 2. The discontinuity sets up a local dipole. The strength of this dipole is proportional to the field of each pole times the separation of the poles. For tight cracks such as SCC the separation is small, hence the dipole is weak. The leakage from the dipole can be detected as long as the sensor is close to the defect. Detection of cracks using in-line inspection works best for cracks that are open to the internal surface. For cracks on the external surface, the crack must be deep so the dipole is close to the internal surface and wide so the dipole is strong.

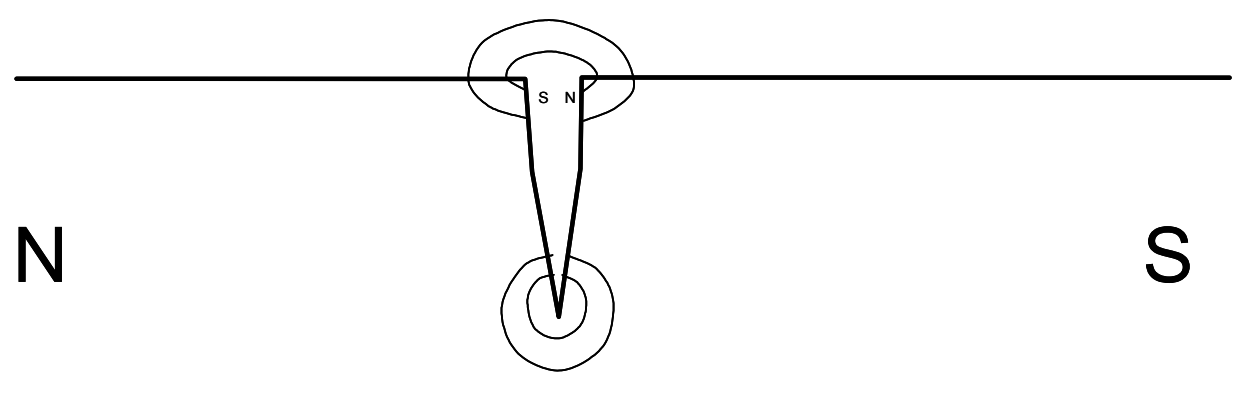

Figure 2. A simple dipole model of a crack in a magnetic field.

Note: The local poles established by the crack oppose the primary magnetizing field. 


\section{Flux Leakage Summary}

For most pipeline flux anomalies, all three models are applicable but one is dominant. For example, flux leakage from corrosion defects is dominated by the metal loss model. However, for larger defects, pipeline pressure causes stress that changes the magnetizing field and the flux leakage. The dipole signal is small for gradual general corrosion. However, for deep pitting corrosion such as that caused by microbes, the dipole signal must be considered, making sizing more difficult.

\section{Magnetic Flux Leakage Tools}

MFL is the most common pipeline inspection technology. Two significant implementations of MFL technologies are axial and circumferential, both of which were used in this project. While the deficiencies of MFL systems are often highlighted, many attributes keep MFL at the forefront of pipeline inspection technologies. Some background material on each is discussed next.

\section{Axial MFL}

Axial MFL is the oldest and still most common implementation of this inspection method. The term axial, describing the orientation of the magnetizing field, was added only recently, after other implementations were developed. Figure 3 shows the typical design of an MFL tool. MFL, without any adjectives, almost always refers to the axial implementation.

Axial MFL has many attributes for pipeline inspection. Inspection speed produces minimal effects for speeds up to $2.5 \mathrm{~m} / \mathrm{s}(5.6 \mathrm{mph})$, and the effects are reported to be manageable for usually larger diameter tool for speeds up to $4 \mathrm{~m} / \mathrm{s}(9 \mathrm{mph})$. Some tools have a minimum speed on the order of $1.0 \mathrm{~m} / \mathrm{s}(2.2 \mathrm{mph})$ for some sensor types and in low-pressure gas lines, below 27.5 bar (400 psi) since significant velocity variations would have to be expected. MFL can inspect with generally the same performance capability for pipe diameters greater than $300 \mathrm{~mm}$ (12 inches). Inspection systems are available for smaller pipeline diameters, as small as $100 \mathrm{~mm}$ (4 inches), with particular reduced performance capabilities because of physical constraints.

While some early MFL systems used electromagnets to magnetize the pipe, most tools now use very strong permanent magnets. These systems have the capability of supplying sufficient magnetic field to saturate pipe material for thicknesses on the order of $30 \mathrm{~mm}$ (1.2 inches) for pipe diameters greater than 24 inches. For smaller pipe diameters, the space available for magnets is constrained and maximum pipe thickness for full saturation will be less. Full saturation is desired to reduce the effects of material variation and stress. At these magnetization levels, detailed loss sizing methods are not significantly influenced by differences in the pipe magnetic properties. Axial MFL systems provide uniform magnetic field at each sensing element. The combination of high strength, uniform magnetic fields, and low velocity minimizes signal conditioning and compensation requirements in the analysis process. 


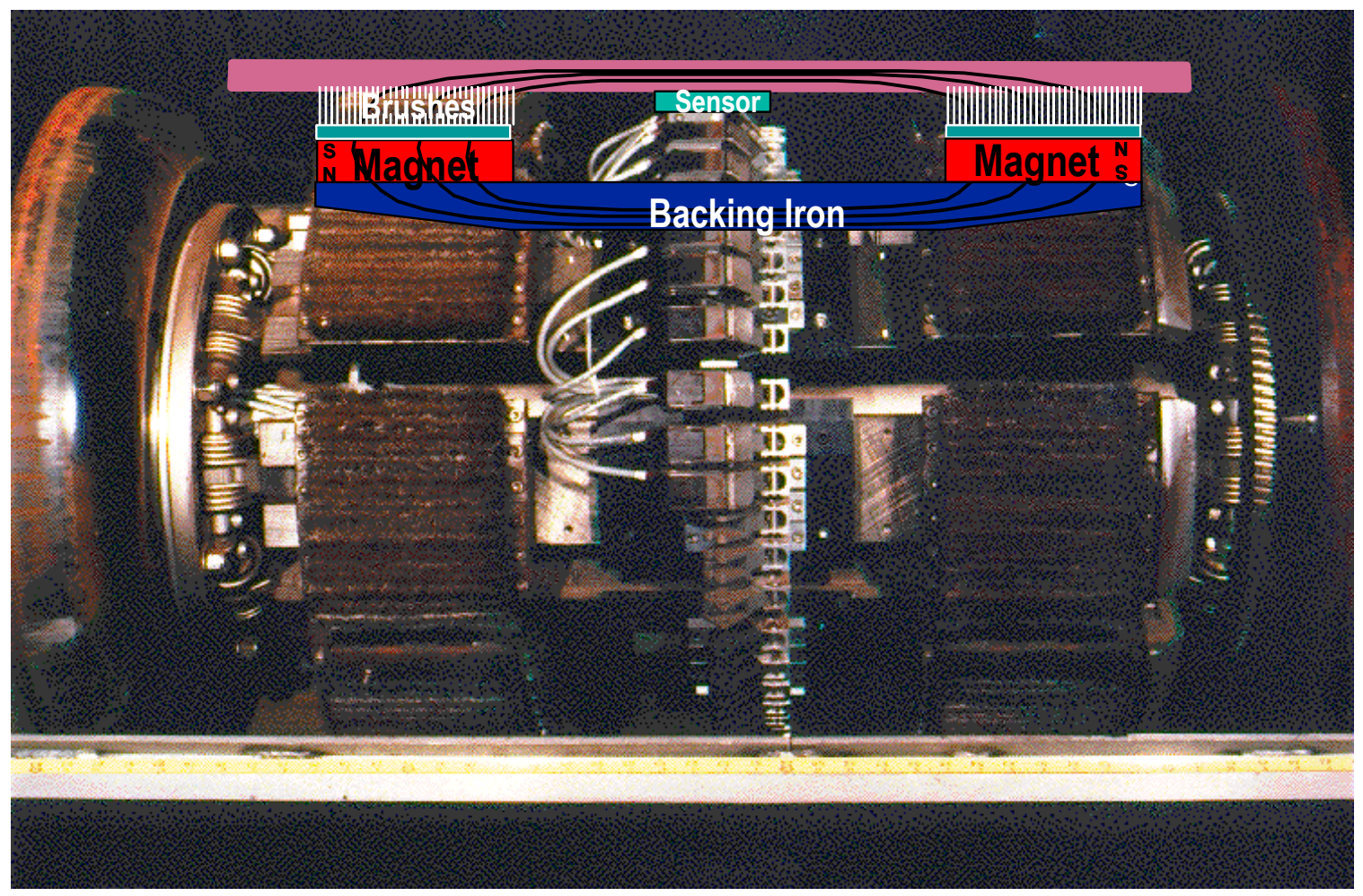

Figure 3. Typical axial MFL tool design.

Many MFL inspection systems are advertised as high resolution. Early MFL systems that stored data on modified 24 channel audio tape recording equipment. High resolution was initially defined as more sensors than one of these early systems. A more scientific definition involves the circumferential extent of defects that need to be sized. Applying the Nyquist sampling theorem to the flux leakage from small pit defects, the sensor spacing must be less than half the circumferential extent of the pit to be assessed. Wall thickness also enters into the sizing accuracy. Pits less than 2 times the wall thickness are more difficult to size because, for defects less than a wall thickness in diameter, most of the flux will spread circumferentially around the defect rather than leak radially from the pipe. Hence, the theoretical sensor spacing for a highresolution tool is on the order of the wall thickness. Since MFL tools must cover a range of wall thicknesses, the practical definition of a high-resolution tool is between 6 and $12 \mathrm{~mm}(0.25$ to $0.5 \mathrm{inch})$. Sensor spacings less than $6 \mathrm{~mm}$ ( $0.25 \mathrm{inch})$ do not provide any significant advantage in defect sizing, though they may be better suited for detecting pinhole defects.

Flux leakage is a vector quantity, and three components can be measured. One sensor can measure one component, and most tools only measure one component, typically the tangential component in the magnetization direction. For axial MFL, the axial component is commonly measured; for circumferential MFL, the circumferential component is commonly measured. The other components, radial and tangential component orthogonal to the magnetization direction, provide additional information that is useful for sizing. Data storage and power restrictions must be considered when adding additional components for sensing and recording. First, a common high-resolution criterion of two sensors spanning for the smallest defect should be met before considering additional components. 
The information from multiple components is correlated. In experiments performed at Battelle's Pipeline Safety and Reliability Center (PSRC) on patch corrosion defects, two sensors components did not provide twice the information; rather the information increase was about twenty percent. For clarification, the benefits of circumferential sensors on an axial tool are quite different than circumferential sensors on an circumferential tool. Combining axial magnetization and sensor data with circumferential magnetization and sensor data has great potential to improve metal loss sizing accuracy.

\section{Circumferential MFL}

Circumferential MFL is a new twist on an old technology. Longitudinal defects, such as SCC, longitudinal corrosion, long seam defects, and axially oriented mechanical damage are of significant concern to the pipeline industry. These defects tend to increase stress levels in the remaining pipe material. To improve detection and sizing longitudinal defects, the orientation of the magnetic field is changed from the traditional axial direction to circumferential. Figure 4 shows a schematic and typical circumferential MFL tool design. While it is feasible to implement circumferential MFL, many implementation variables must be considered.
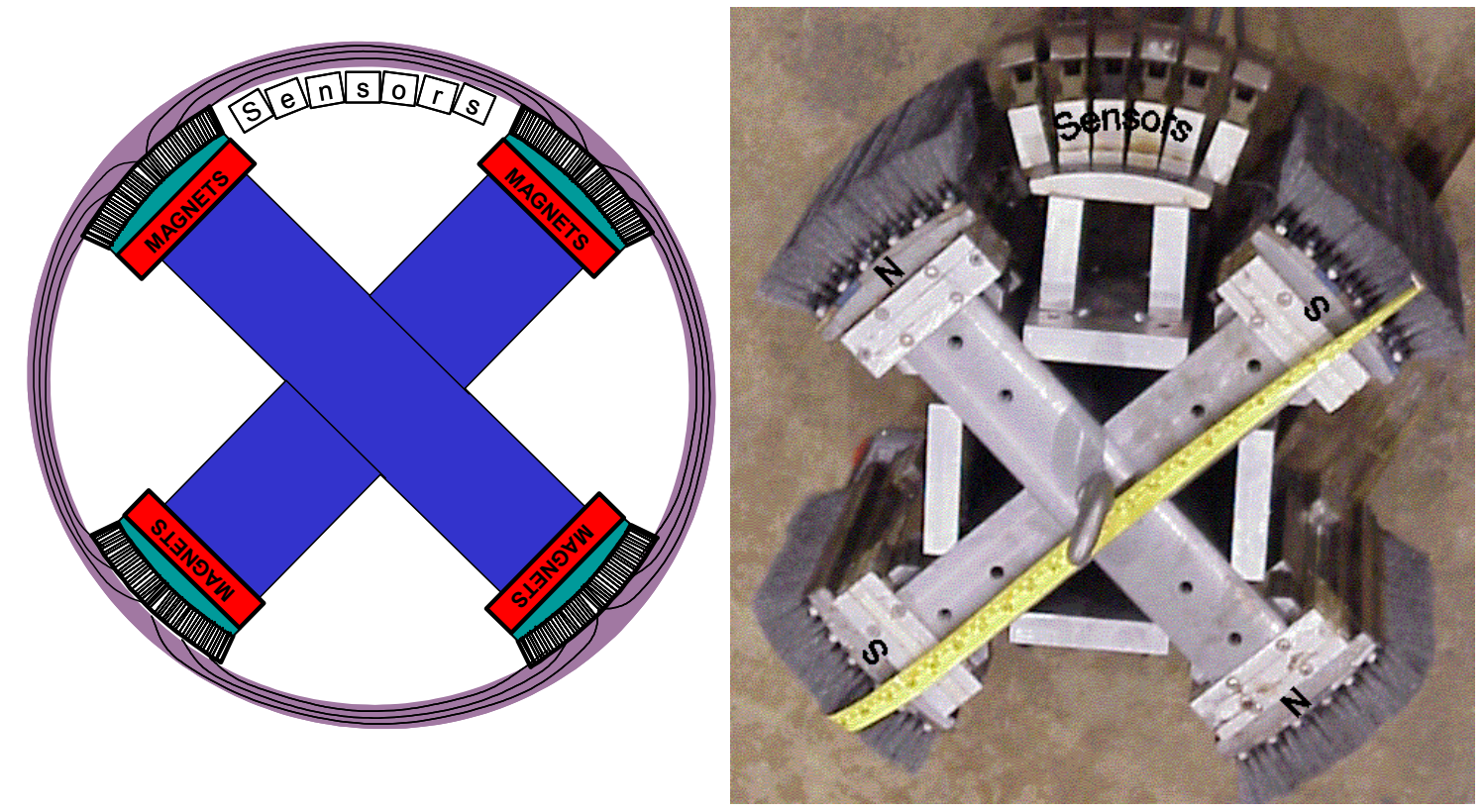

Figure 4. Schematic and typical execution of circumferential MFL tool design.

Magnetization is a significant inspection variable, since it is much more difficult to saturate the pipe material in the circumferential direction. In situ magnetic measurements, provided in Figure 5, show that permeability in the circumferential direction is a quarter of the axial permeability. Magnetic finite element modeling can be used to show the pipe material near the magnetizer pole is saturated (permeability $\sim 15$ ) while the material midway between the poles is able to carry more flux (permeability $\sim 75$ ). A nearly linear relationship between applied field and flux density exists, indicating that the circular geometry of the inspection method will limit improvements made by magnet strength improvements and new magnetizer designs. 
June 2003

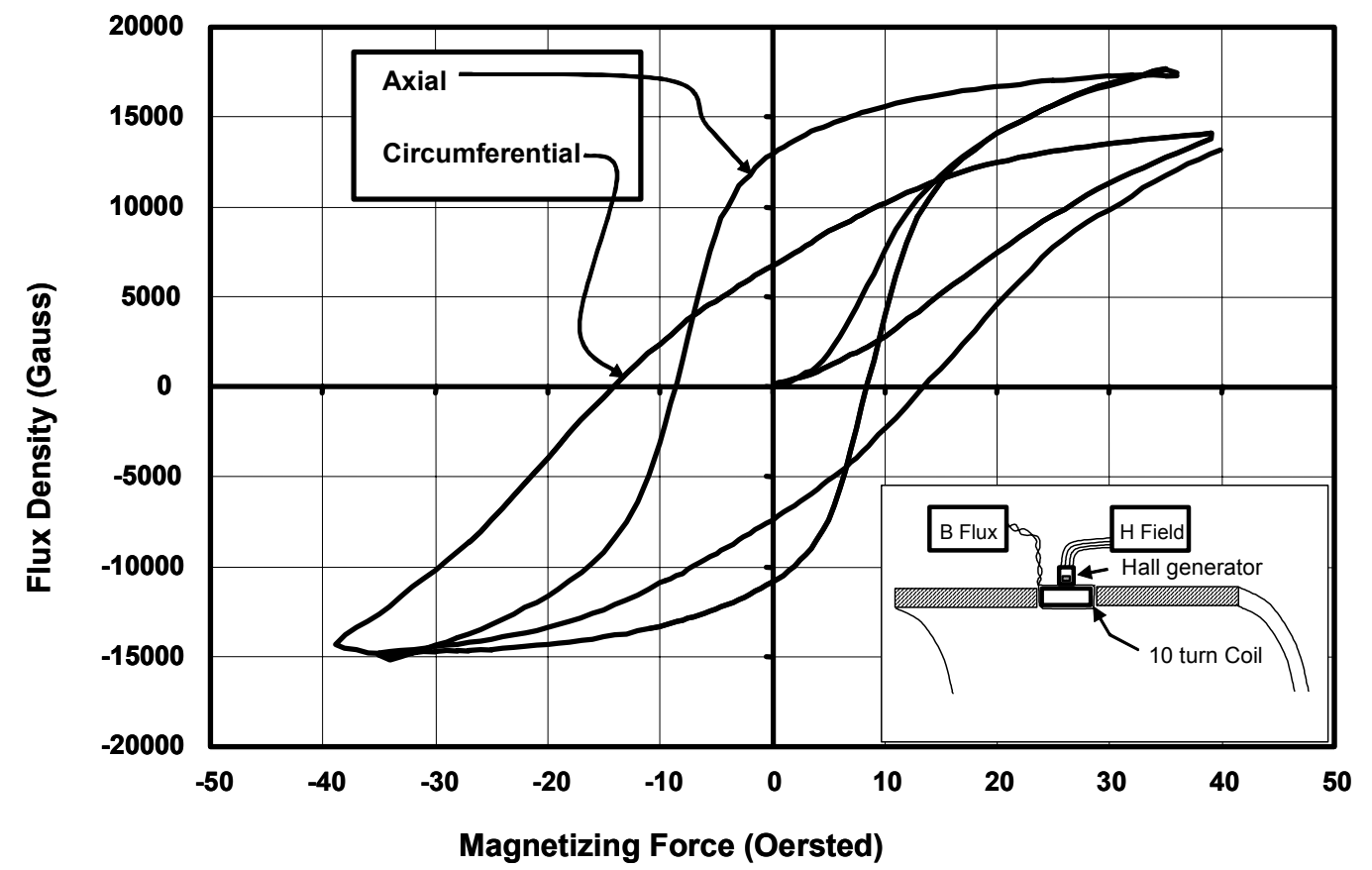

Figure 5. In-situ magnetic measurements demonstrating difficulty in saturating the pipe material in the circumferential direction.

As shown in Figure 6, the strength of the magnetic field is greatest near the magnetizer poles and smallest at the center. This is caused by the poor magnetic properties of the pipe material in the circumferential direction and the circular geometry providing alternative flux paths. This nonuniform field makes defect sizing more difficult. A defect near one magnet pole gives a different signal than the same defect equidistant from magnets. Compensation methods are used to adjust the amplitudes to an average magnetization level. However, compensation for magnetization level adds a significant amount of complexity to defect detection and analysis procedures. For defects that span the poles, the compensation may mask the defect signal, resulting in a false dismissal or the undersizing of a potentially critical defect.

Velocity effects for circumferential MFL are detectable at lower speeds and are more significant than for axial MFL. Velocity induced signal changes are detected at speeds of $1.0 \mathrm{~m} / \mathrm{s}(2.2 \mathrm{mph})$. If the defect is midway between the poles, the amplitude is simply reduced. However, if the defect is closer to one pole, the flux leakage is also distorted. At inspection speeds of $1.0 \mathrm{~m} / \mathrm{s}$ (2.2 $\mathrm{mph}$ ), the magnetizing field starts to concentrate at the inner surface of the pipe, making detection of OD defects more difficult.

In summary, the orthogonal magnetization direction of circumferential MFL overcomes some of the weakness of axial MFL for detecting axial defects. However, circumferential MFL has weaknesses itself. Defect location relative to a pole, magnetization level, velocity, and sensor spacing are inspection variables that must be considered. These factors make implementation and analysis more difficult, thus reducing many performance gains achieved by changing the orientation of the magnetizer. 


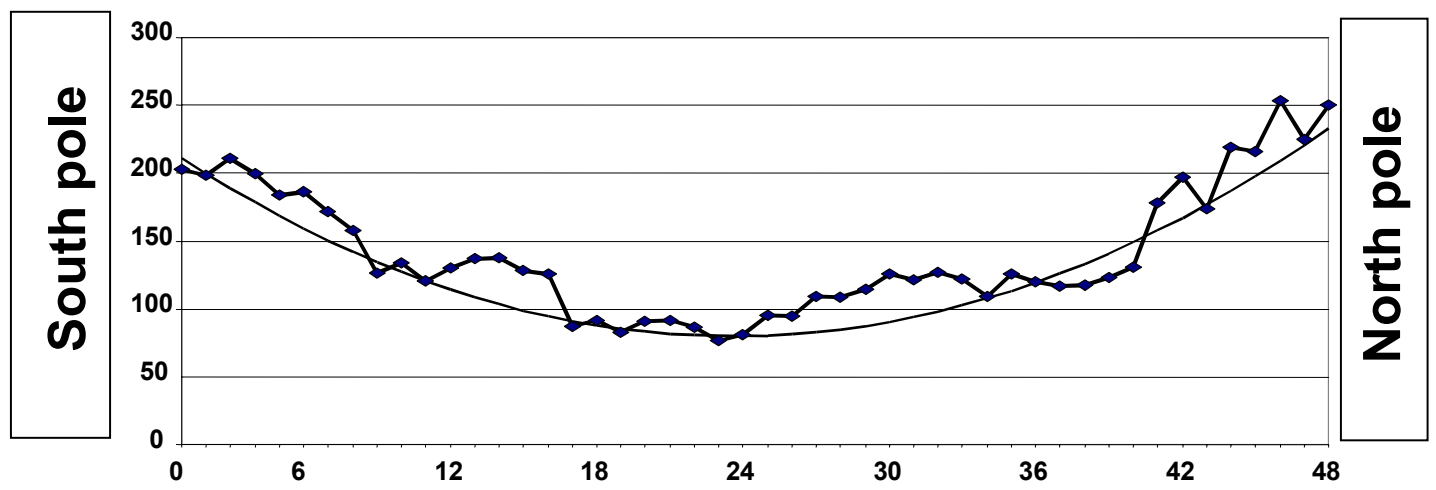

Figure 6. The strength of the magnetic field is greatest near the magnetizer poles and smallest at the center.

\section{Prior Circumferential MFL Developments}

The earliest attempts at implementing a circumferential MFL system were made by Tuboscope in the 1970s. At that time, coil sensors were used on all MFL tools, including the Tuboscope circumferential tool. The Tuboscope coils required circumferential movement to produce a measurable signal. As a result, Tuboscope built the tool to rotate as it moved down the pipeline. The concept was sound, but it proved too difficult to implement, so Tuboscope eventually abandoned the idea.

The Pipeline Research Council International (PRCI) began investigating circumferential MFL in 1994 [9]. This early work concentrated on the feasibility of using circumferential MFL to detect cracks using a laboratory-scale mockup. As discussed in later sections of this report, feasibility was demonstrated.

A commercial inspection tool that uses circumferential MFL was developed in the mid to late 1990s by GE (formerly Pipeline Integrity International, which was formerly British Gas). The tool was successfully used to detect narrow axially oriented corrosion in a pipeline where the spiral wrapped protective coating tented at the girth weld. The long narrow tent allowed moisture to collect and axially long, circumferentially narrow corrosion defects to form.

According to company literature, the new circumferential MFL tool successfully detected narrow axially oriented corrosion and it sized them with sufficient accuracy. GE reports a second successful application of circumferential MFL, in which the tool detected hook cracks on the inside surface of an ERW pipe.

In 2000, Battelle began a program to evaluate the use of circumferential MFL to detect and size mechanical damage. This program includes fundamental studies of magnetization and leakage fields and the effects of inspection parameters such as tool velocity and defect location (inside or outside diameter). It also includes studies of many tool design issues, such as magnetization strength, pole length, and pole width. 
Throughout all the work described above, the detection of all cracks of significance in the body of the pipe with circumferential MFL has remained elusive. Feasibility was demonstrated in the 1994 PRCI program, and basic studies on circumferential MFL fields are under way in a current Battelle program. In addition, practical experience is being gained by inspection tool vendors.

\section{Other Developments Aimed at Detection of Axial Cracks}

In addition to the circumferential MFL developments described above, a number of attempts have been made to detect cracks using other inspection techniques. Previously developed nondestructive testing techniques (including angle beam ultrasonics, electromagnetic acoustic transducers, and remote field eddy current) have proven capable of detecting longitudinal cracks.

GE developed the first angle-beam ultrasonic tool in the 1970s, and the tool has seen some use in operating pipelines. The tool used liquid-filled wheels to allow ultrasonic energy to be coupled into the pipe wall of gas-filled lines. The number of sensors is limited by the number of wheels, and sophisticated signal processing is needed to detect and characterize cracks. Generally, the tool has proven effective at detecting and sizing some cracks and crack colonies. Questions about its ability to discriminate between crack signals and non-crack signals remain. In addition, reliable estimates of probabilities of detection and sizing accuracies are not available.

Pipetronix (now part of GE) developed an angle-beam ultrasonic tool in the 1990s, and the tool has also seen use in operating pipelines. This tool operates only in liquid-filled lines. The liquid couples the ultrasonic energy into the pipe wall, negating the need for a wheel. The Pipetronix tool has many more sensors than the wheeled tool, and it is reported to have greater sizing and detection accuracies.

Several attempts have been made to develop a commercial electromagnetic acoustic transducer (EMAT) inspection system for cracks. Early efforts directed at pipeline inspection included work by C.W. Pope in Australia and T.D. Williamson in the United States in the 1980s and 1990s. These efforts were combined in the middle 1990s, then transferred to Tuboscope in the late 1990s. The tool is still under development.

Finally, work has also been done on the use of remote field eddy current and velocity induced eddy current techniques to detect and size cracks. The most successful of these efforts has been using remote field techniques. Significant restrictions exist on the velocity at which the techniques can be used. Both DOT and DOE are funding separate developments in remote field technology.

None of the ultrasonic or eddy current techniques described above has proven widely successful. Each has limitations with applicability, such as a minimum pipe diameter, maximum inspection speed, or operating medium. Furthermore, the complexity of the systems makes the cost of an inspection higher than a corresponding MFL corrosion inspection. 


\section{Prior Research Results Using CirCumferential MFL}

\section{Stress Corrosion Cracks and Weld Corrosion}

\section{SCC - Unstressed Conditions}

The potential of circumferential MFL to detect longitudinal defects in the absence of stress was demonstrated by a PRCI program [9] in which an external magnetizer was used to apply a circumferential magnetic field to a pipe segment containing stress-corrosion cracks. Figure 7 shows the results of a magnetic particle inspection of the outer pipe surface of a pipe sample; cracks appear as horizontal lines colored red or yellow in the photograph.

Figure 8 shows results from the circumferential MFL inspection. Flux leakage appears as dark regions in the center plot. Crack profiles through the thickness are shown in the call outs. The signals from one large crack near the top of the plot and two neighboring smaller cracks near the middle and bottom of the plot indicate that circumferential MFL could detect cracks. The signal from the middle crack is not strong, though, and it could easily have been missed in a field application.

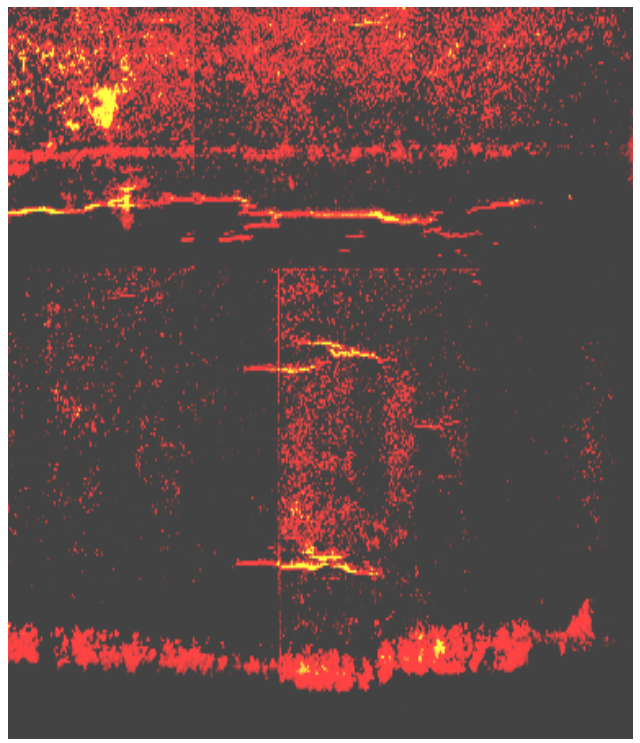

Figure 7. Results of a magnetic particle inspection of the outer pipe surface.

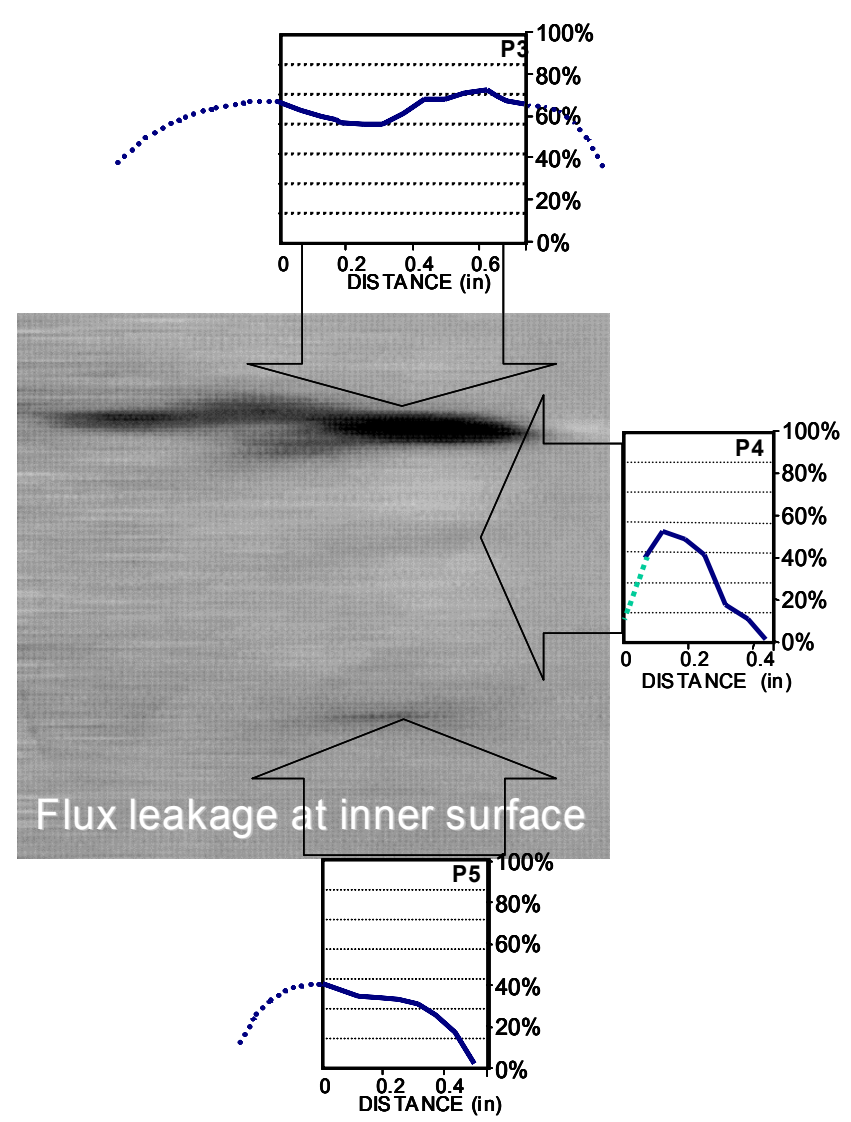

Figure 8. Circumferential MFL results. 


\section{SCC - Stressed Conditions}

Stress is known to impact magnetization levels; consequently, the concept of a stress-enhanced magnetic flux leakage signal was also examined in the PRCI program [9]. Circumferential flux leakage signals were acquired on a pipe sample while internal pressure was applied. Images of stress corrosion cracks were acquired at 0,250 , and 500 psi internal pressure in a 30 -inch diameter, X52 pipe with a wall thickness of 0.375 inch. Conservative calculations showed that this defect could fail at pressures as low as 800 psi.

The experiments showed that internal pressure greatly reduces the applied magnetic field levels. To enable crack signals to be compared at different pressures, field levels were adjusted using electromagnet augmentation to attain a 30 Oersted field level at each pressure. Figure 9 shows three images of signals from the stress corrosion crack region. The top image is the crack at pressure $500 \mathrm{psi}$, the middle image is the crack at no internal pressure, and the bottom image is the difference between the signals at high pressure and no pressure. These plots are similar to the plot in Figure 8 except that the signals are shown in color rather than black and white. In the figures, the maximum signal in each case is shown in red, and the magnitude of the signal can be seen in the legends shown at the right.

Figure 9 shows that at high pressure the signal levels increase approximately 12 to 14 gauss or 20 percent. These results show that signals from stress-corrosion cracks can be augmented by internal pressure, provided that this pressure locally increases the stress in the pipe wall. The fact that significant stress effects would only be detected at pressures that begin to threaten integrity suggests an alternative concept for pipeline inspection.

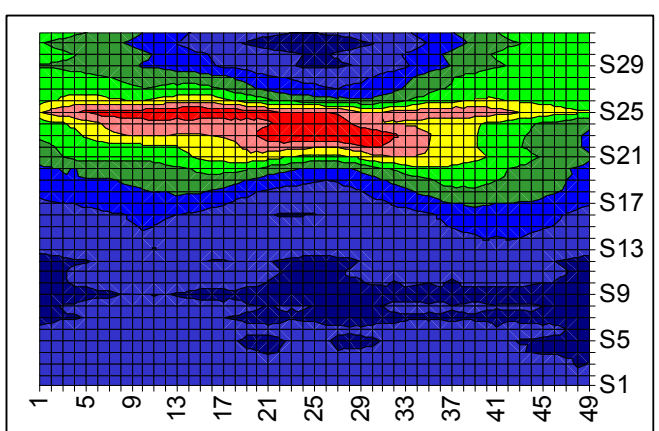

500 psi
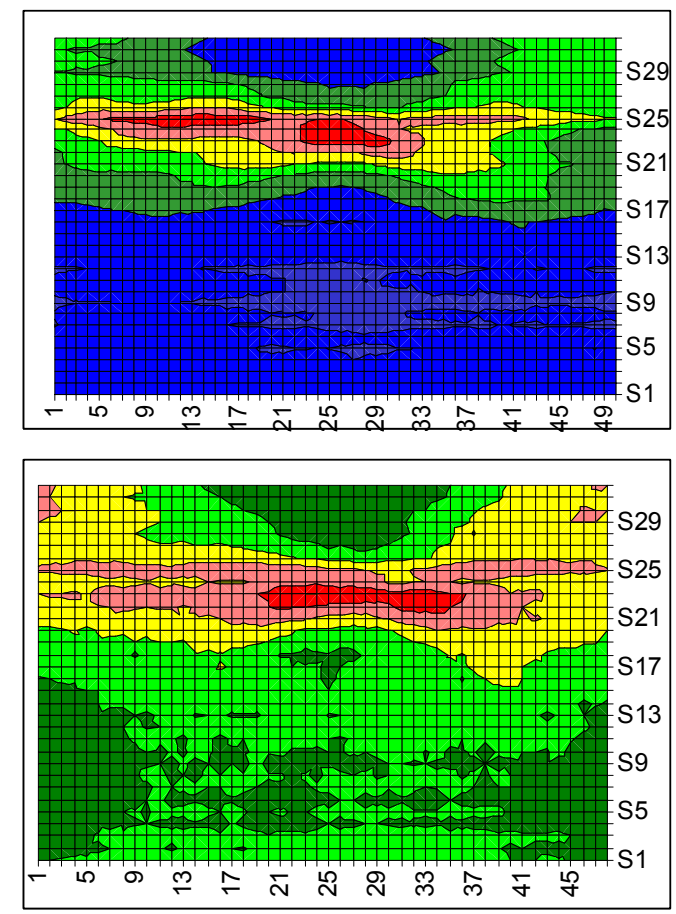

\section{Difference}

$\square$ 12-14

$\square$ 10-12

$\square$ 8-10

$\square 6-8$

$\square$ 4-6

$\square 2-4$

$\square 0-2$

Figure 9. Circumferential MFL signals under pressure. 


\section{Seam Weld Corrosion}

Circumferential MFL was also used to examine selective seam weld corrosion on an electric resistance welded (ERW) sample. With the seam weld examinations, the weld itself always gave a signal. So, changes in the signal were used to indicate where corrosion may exist. Signals from good welds, welds with general corrosion, and welds with selective seam weld corrosion are shown in Figure 10. In this figure, the signals are shown as a cross section of the circumferential leakage field.

Figure 10 shows strong signals are possible from selective seam corrosion in ERW pipe. In addition, nearby metal-loss corrosion is also visible. When both selective seam corrosion and metal-loss corrosion occur, the signals overlap, which confuses their interpretation. Based on these results, circumferential MFL has the potential for detecting selective seam weld and metal loss corrosion; however, many implementation and signal interpretation problems remain.

\section{Effects of Stress on MFL Signals}

The MFL can also be used to measure changes in the magnetic properties of the pipeline being inspected. Stress affects the magnetic properties of magnetic materials such as pipeline steels. These magnetic changes caused by stresses, albeit sometimes subtle, will produce flux leakage fields that can be detectable if proper measurement techniques are employed. MFL measures a volumetric average of the magnetic deformation. Therefore, exact information on the residual stresses, plastic deformation, and cold work within a defect cannot be obtained. Even so, the MFL technique can provide valuable information about mechanical damage defects [7].

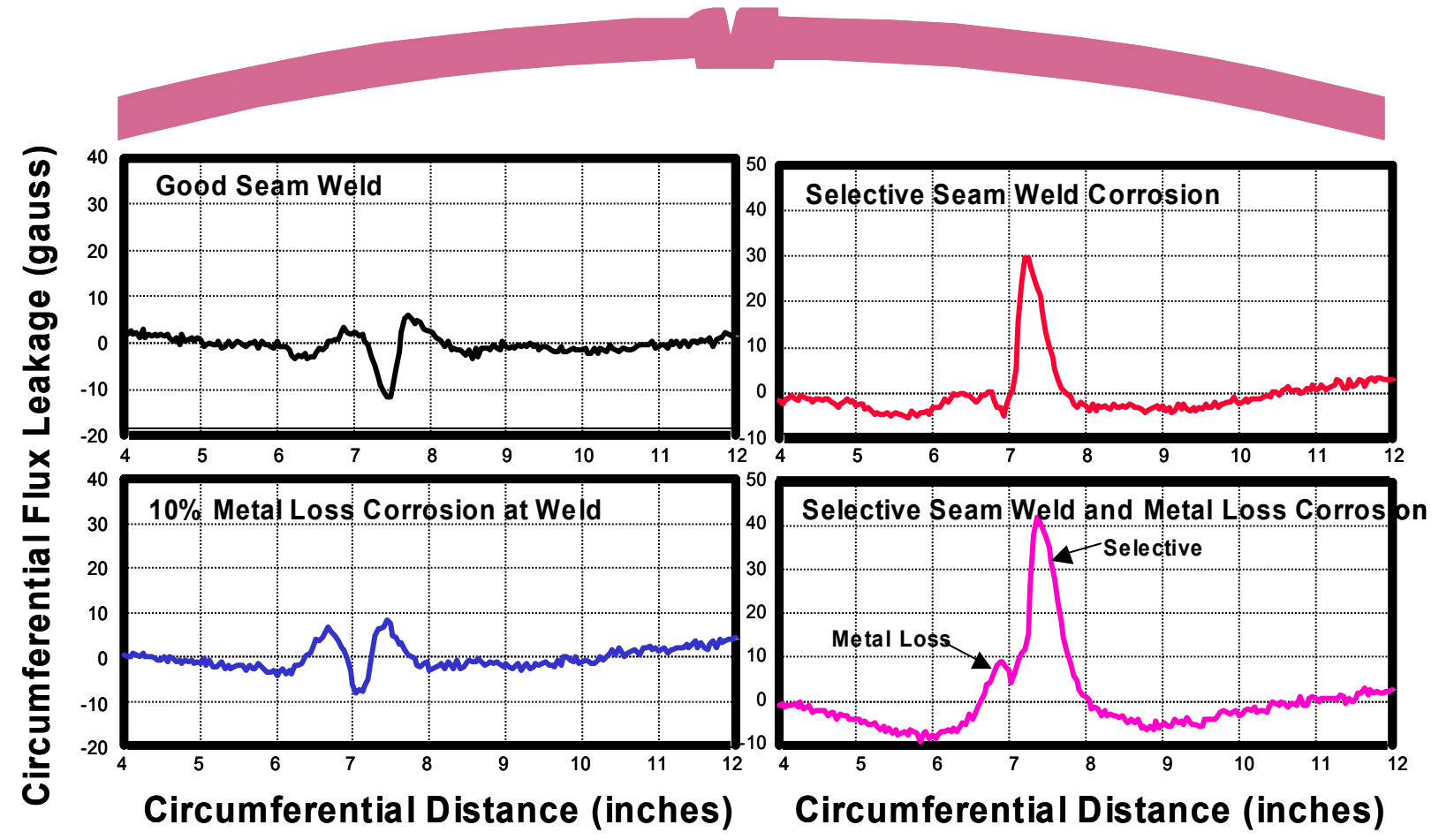

Figure 10. Circumferential MFL signals from selective seam corrosion. 
The effect of compressive residual stresses is illustrated in Figure 11. At the left of Figure 11, the curves show that the magnetization is nearly independent of field levels A zone of compressive stress was created by compressing the surface of a pipe with a force sufficient to cause a few percent plastic deformations. Inspection using a magnetization level of 110 Oersted, a level at the low range of commercial MFL tools, produced a leakage of less than 10 gauss. Using a lower magnetization field to 70 Oersted causes a larger signal of 30 Oersted. This illustrates that lower magnetic field are more sensitive to stress effects.

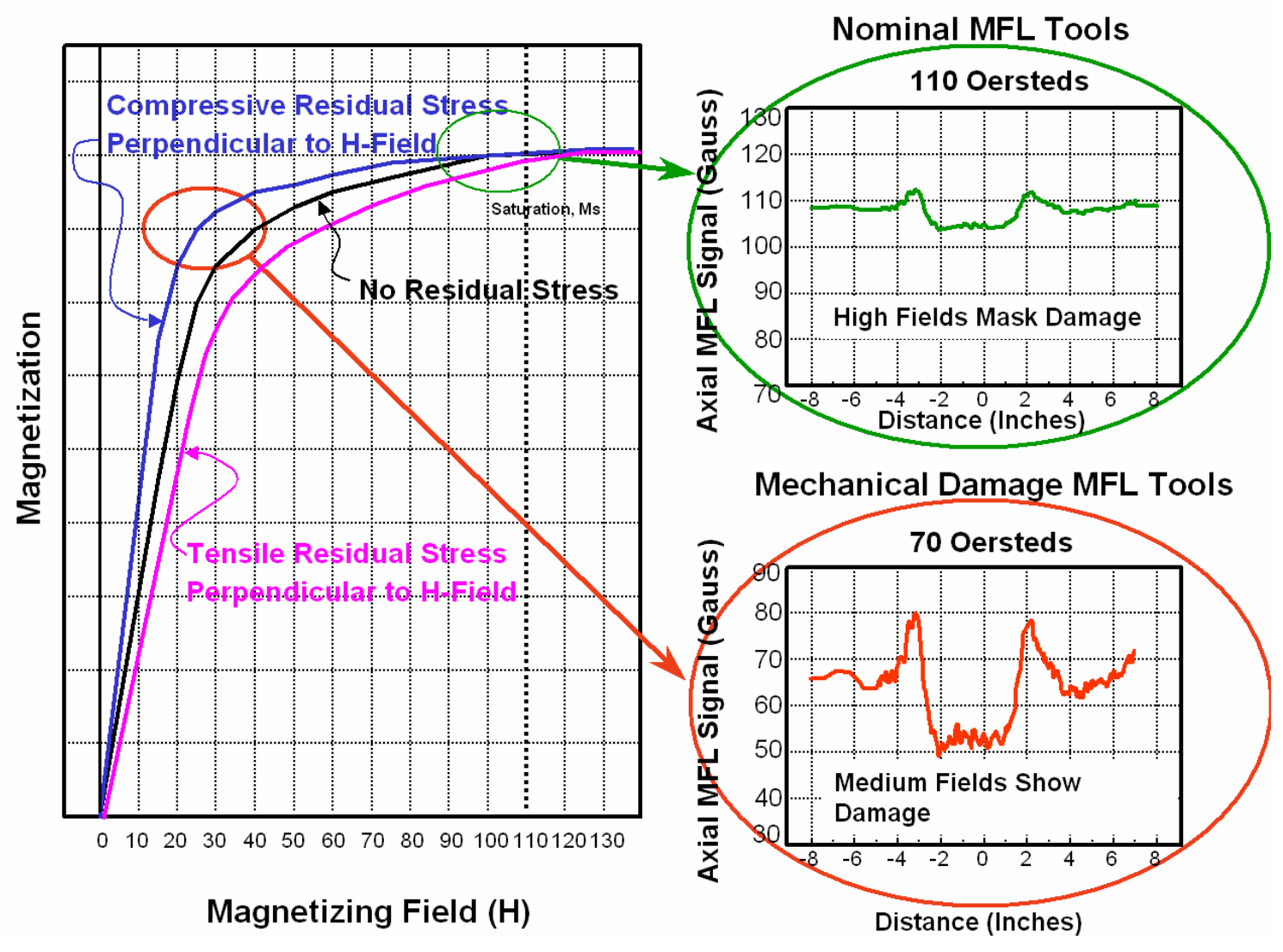

Figure 11. The effect of compressive residual stresses. 


\section{EXPERIMENT PROCEDURE AND EQUIPMENT}

The goal of this project is to improve MFL technology for SCC detection and sizing, building on previous research results and equipment. While MFL systems are often criticized for resolution deficiencies, MFL technology can overcome the physical and practical inspection challenges presented by transmission pipelines. MFL will be used for many decades and improved inspection results will enhance pipeline safety while helping ensure product delivery.

Two approaches to improve MFL systems were taken. The first involves detection of local areas of high stress at crack tips. A circumferential MFL implementation that enabled testing at multiple magnetization levels was used. The circumferential magnetizer orients the field orthogonal to the crack. High and low magnetization levels can be used to detect stresses. The second approach examined the combination of axial and circumferential implementations. The axial data, while not effective for crack detection, has potential for noise reduction and signal identification to distinguish cracks from volumetric defects.

The experiments used existing defect sets, previously developed equipment, and new components. Where appropriate, this project used equipment that is part of the Pipeline Safety and Reliability Center (PSRC). The PSRC supplied both natural SCC samples and pipe with artificial flaws. A new circumferential MFL tool including sensors was built that uses a PSRCsupplied MFL data recording module. To conduct the tests under pressure, a special apparatus was designed and configured that used PSRC Flow Loop compressors to provide the highpressure gas. The details of the research apparatus used to conduct the experiments are provided next.

\section{Test Configuration}

To evaluate leakage signals from cracks under stress, a method was established to acquire flux leakage at multiple pressures repeatedly at multiple magnetization levels. The experimental configuration is shown in Figure 12. The components include:

- A pipe sample with defects configured with flanges on either end (PSRC sample).

- A pig launching barrel for insertion of the circumferential magnetizer and data recorder. This was simply a half-inch diameter $60 \mathrm{ksi}$ yield pipe from the PSRC.

- A hinged pressure door for insertion and access to the magnetizer and data recording equipment.

- Rods for pulling the magnetizer and data recording equipment in either direction.

- Rod seals to hold pressure as the equipment is pulled. These seals are the same used in oil well pumping operations.

- A pressure relief valve to prevent over pressurizing. This was required since the stress at the notch tip is near yield. 
June 2003
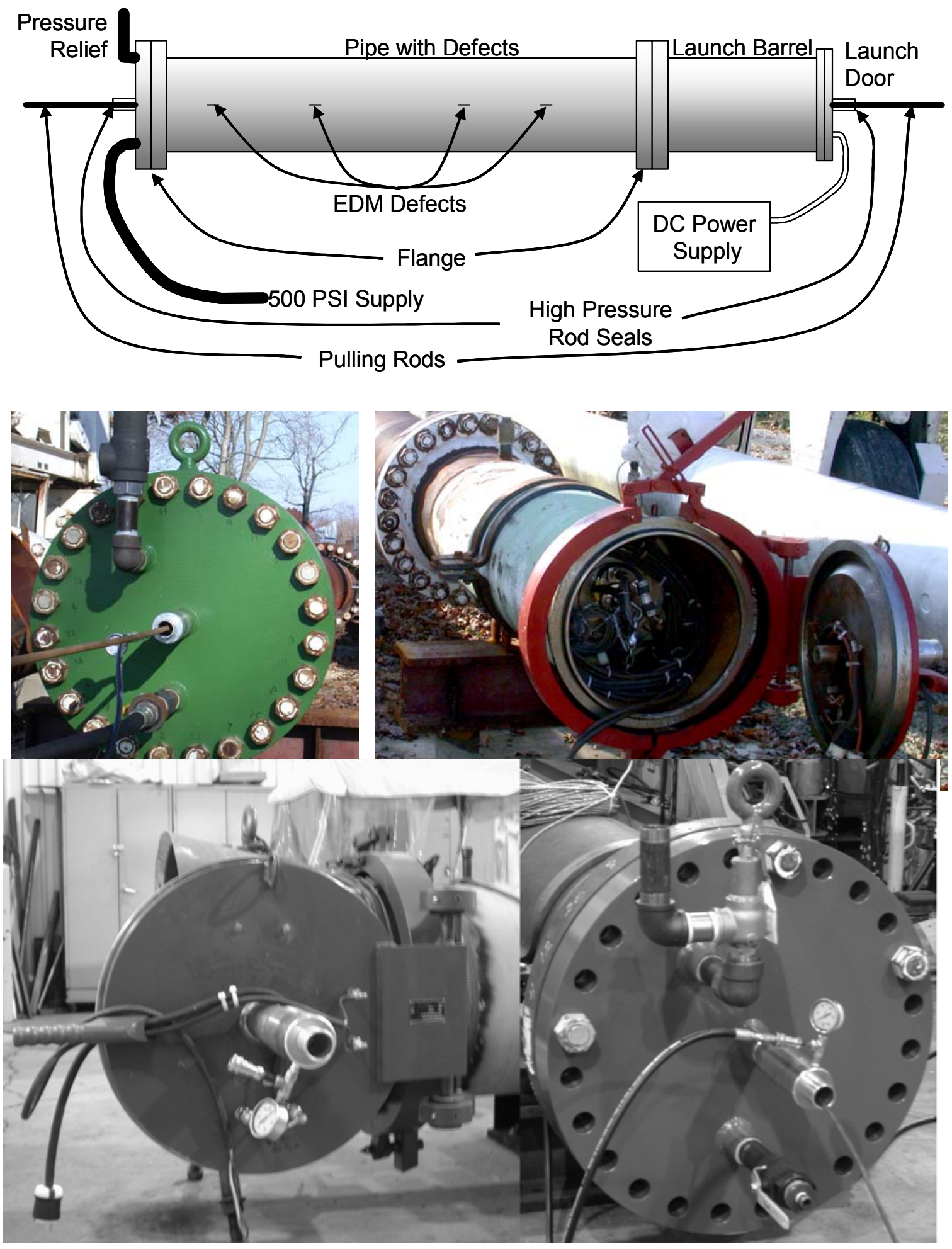

Figure 12. Apparatus to acquire data at multiple pressures and multiple magnetization levels. 


\section{DEFECT SAMPLES}

Two pipe samples from the PSRC were used. The sample used in pressure tests had simulated cracks. The sample that was examined using both axial and circumferential MFL had natural SCC. Details of these two samples are provided next.

\section{Artificial Crack Sample}

The artificial crack sample was prepared for the PSRC by SwRI in 1997. This 40-foot sample has a wall thickness of 0.344 inches and $70 \mathrm{ksi}$ specified minimum yield strength (SMYS). It has been used for testing of crack detection pigs including ultrasonic and electromagnetic acoustic transducer (EMAT) sensors. This sample has both electro discharge machining (EDM) notches and weld deposition cracks. Figure 13 shows examples of both types of cracks. The EDM notches were 0.020 inches wide and had lengths ranging from 0.5 to 4 inches (12 to $100 \mathrm{~mm}$ ). The weld deposition cracks are installed using the following procedure:

1. A shallow axial groove (width $=1 / 8^{\prime \prime}$ ) is ground in the pipe surface. The depth is typically 0.125 less than the desired depth of flaw.

2. Notching the sample to a depth of five percent of desired depth

3. Adding a material that promote cracking of the parent material

4. Welding over the notch with material that closely matches the chemical composition of the pipe.

As the weld cools, cracks form in the weld material and propagate into the pipe. Excess material is removed by grinding. This process is used to produce calibration samples to assess nondestructive testing methods for nuclear reactor piping. This technique is well accepted in the nuclear industry, but it should be noted that ultrasonic methods are the primary inspection technology.

The artificial cracks are in three rows, two in the parent material and one in the heat affected zone of the seam weld. The seam is a double submerged arc weld and defects were introduced on either side of the weld bead. The configuration of the notches and cracks are identical in both the EDM and weld deposition samples. The depths of these defects were evaluated using phased array ultrasonic methods.

In order to examine the effect of stress from planar defects, data were acquired from near narrow EDM notches at notch-tip stresses approaching specified minimum yield stress (SMYS) of the material. To ensure stress levels were near SMYS levels, finite element modeling methods were used to calculate the stress near the notch tip in the pipe sample. Figure 14 shows that for a 500 psi internal test pressure, the stress 10 percent below the notch exceeds the $65 \mathrm{ksi}$ SMYS of the pipe material. Therefore, the pipe material is approaching yield near the notch tip. The notches were 0.020 inches wide; the mechanical finite modeling showed the notch opening changed less than ten percent at the highest applied internal pressure. 
June 2003

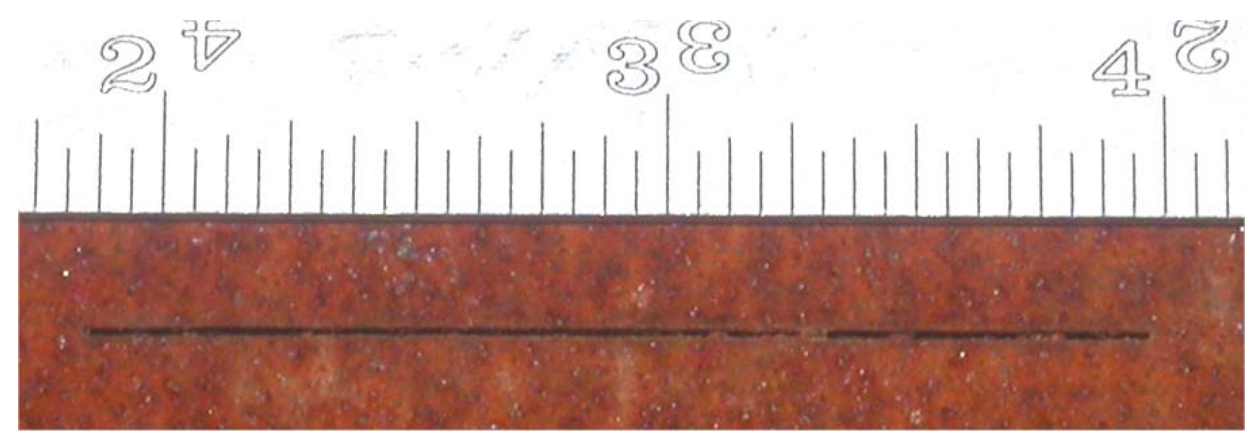

a. Electro-Discharge Machined Notch

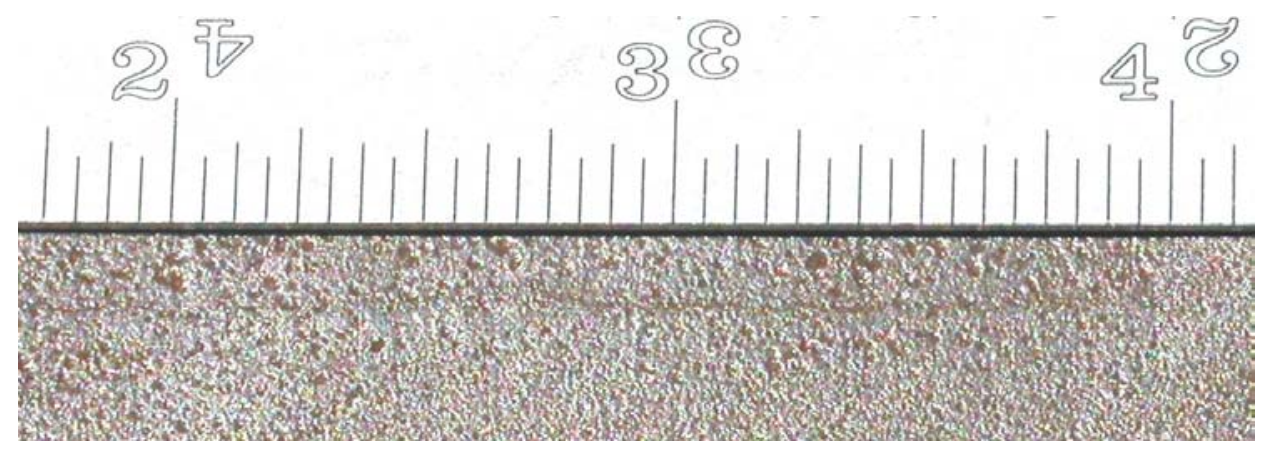

b. Weld Deposition Crack — Photo

$$
G=1.95 \text { "Long }
$$

c. Weld Deposition Crack - Liquid Penetrant Enhanced

Figure 13. Example of EDM notch and weld deposition crack defects. 
June 2003

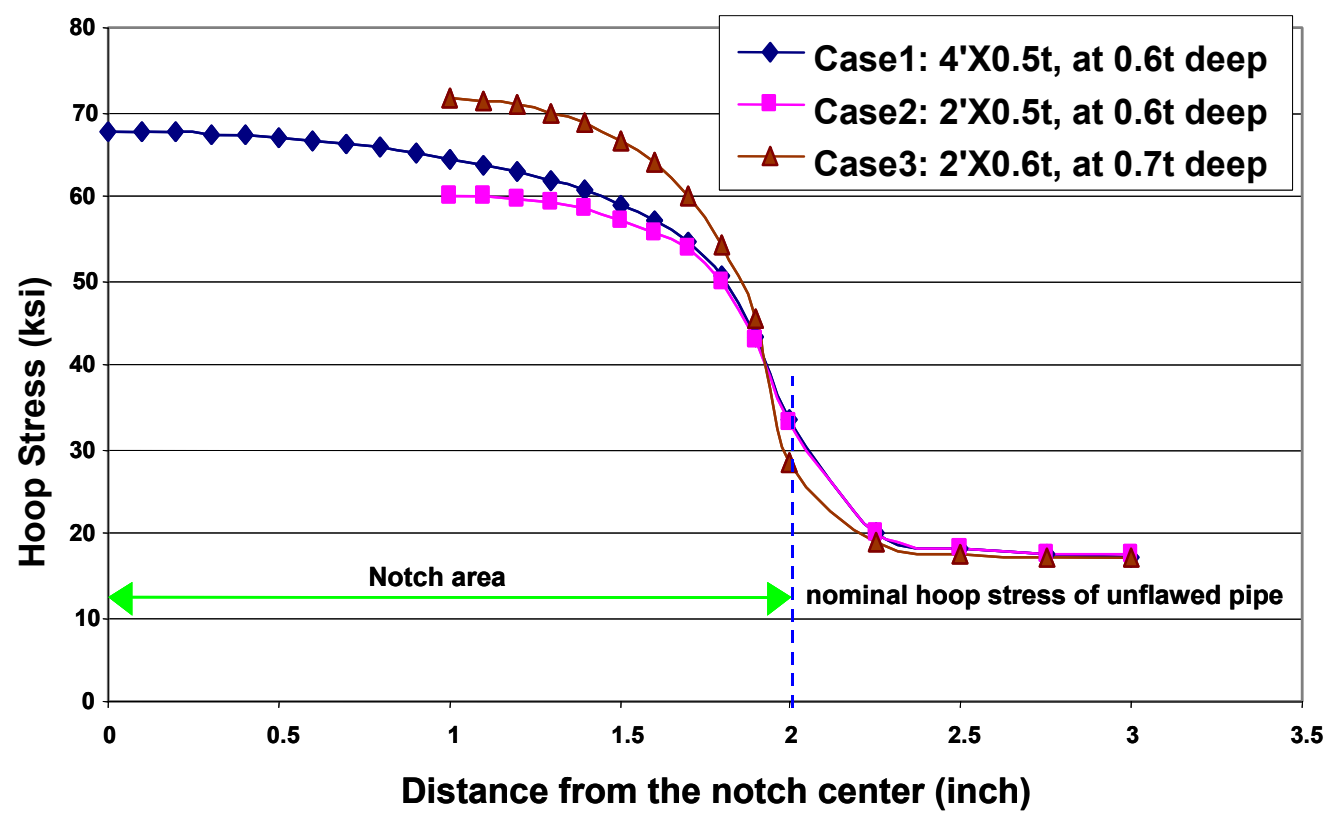

Figure 14. Stress at and near the notch tip.

Note: $\quad$ At 500 psi internal test pressure, the stress under the notch exceeds the $65 \mathrm{ksi}$ SMYS of the pipe material

\section{Natural SCC Sample}

Natural SCC samples in 24-inch diameter pipe have been historically difficult to obtain. However, the PRCI has collected a good supply of natural cracks in both 26- and 30-inch diameter pipe; Battelle stores these samples at the PSRC. For other experiments conducted for the PRCI, a 26-inch diameter sample was reduced to 24 inches in diameter. This 8-foot sample was used on this project for crack detection. To make this conversion, the pipe was split at the seam weld and approximately 6.28 inches of material was removed. The pipe was pulled together and seam was welded manually. While the cracks in this sample are natural, they were more open than usual. The crack zone was located using wet fluorescent magnetic particle inspection. The sample also has a series of corrosion pits that parallel the crack zones about 6 circumferential inches away.

\section{CIRCUMFERENTIAL MAGNETIZER}

While the PSRC had a circumferential MFL magnetizer, this unit could not supply sufficient field to perform high- and low-magnetization testing. This permanent magnet circumferential MFL test bed used magnets from the axial MFL magnetizer that could achieve 150 Oersted field levels in the axial direction. Removing magnets and adding demagnetizing shunts enabled testing at reduced field levels of 50 to 70 Oersted in the axial direction. However, when the magnets were placed in a circumferential configuration, the maximum field levels that could be 
attained were 30 to 50 Oersted depending on pipe material and wall thickness, values that were too low for the high and low field comparisons.

In view of the problem noted above, a new circumferential magnetizer was built specifically for testing stress effects. A variable field level was attained by an electromagnet. While this design is not practical for in-line inspection tool use, it does facilitate the research goals of this project. If the crack detection and sizing capability would be enhanced by using multiple magnetization levels, this electromagnet implementation could be replaced with a permanent magnet design. To attain the highest possible field level for these experiments, a unique magnetizing pole configuration was used. Shown in Figure 15, the configuration has similar poles separated by 60 degrees and a 120-degree sensor region. The effectiveness of this design was confirmed and enhanced using magnetic finite element modeling. It was estimated that 5000 ampere turns would be needed to saturate the pipe material. Each electromagnet pole has 308 turns of 8 gage squared wire. The heavy gage wire was needed to carry the high electrical current. The axial pole length is 18 inches and each pole was 2 inches wide. Brushes 2 inches long were used to center the tool in the pipe, account for pipe dents and weld mismatches, and couple the flux into the pipe wall.
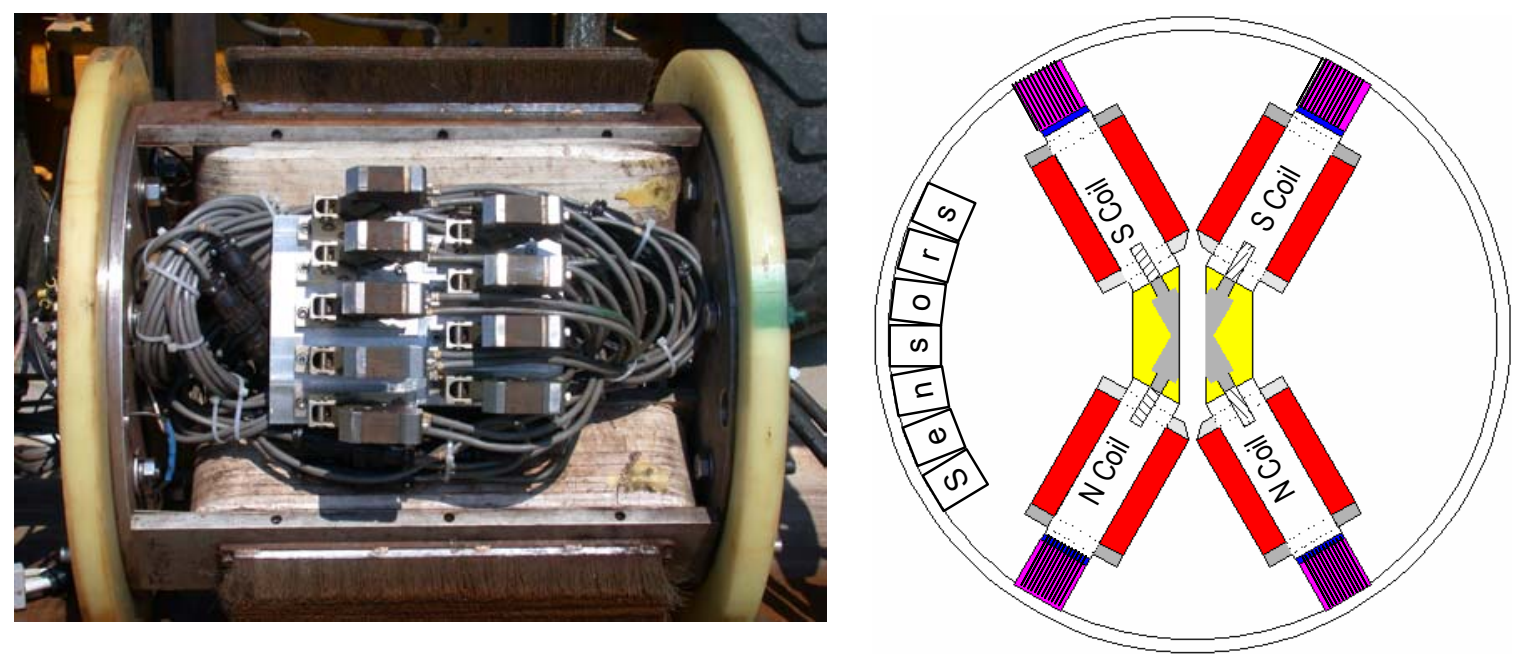

Figure 15. Electromagnet circumferential magnetizer.

A fully configured magnetizer was pulled into a 0.300 -inch wall thickness pipe and magnetic field measurements recorded around the circumference of the pipe using a three axis gaussmeter. Figure 16 shows the test results, where the highest circumferential field occurs between opposite poles and a designated null is present between like poles. However, the field in the pipe between the poles is quite variable. The maximum field level is attained at 10 to 15 degrees from the magnetizing pole and is substantially lower midway between the poles. For the applied magnetizing current of 22 amps, the peak field was 150 Oersted, which would eliminate stress effects, but the field between the poles was 50 Oersted, in the middle of the stress effects zone. At this field level, stress effects would still be seen. 


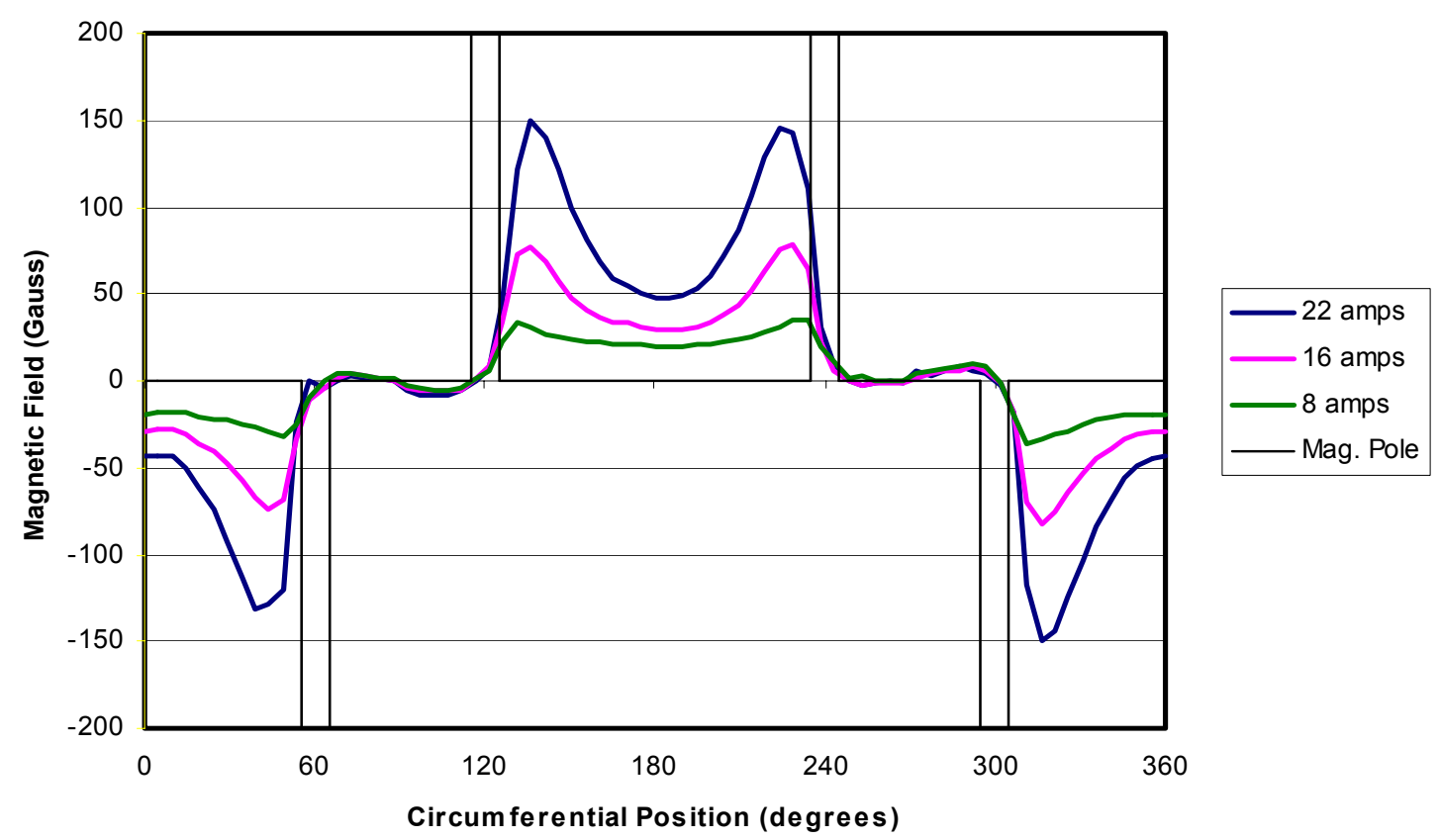

Figure 16. The circumferential component of the magnetic field around the circumference of the pipe for the electromagnet test bed vehicle circumferential magnetizer.

Figure 17 shows the radial component of the magnetic field around the circumference of the pipe. This figure shows a large portion of the flux leaks out of the pipe into the air. The opposing high radial field between the like poles indicates a lower than expected permeability and saturation in the circumferential direction.

The magnetization results shown in Figures 16 and 17 demonstrate it is much more difficult to saturate the pipe material in the circumferential direction. In situ magnetic measurements, shown in Figure 18, indicate that permeability in the circumferential direction is a quarter of the axial permeability. This anisotropy in permeability is most likely due to the pipe fabrication process.

Magnetic finite element modeling was used to visualize the flux leakage in the pipe and surrounding medium. Taking advantage of symmetries, a quarter of the circumferential magnetizer was modeled. The modeling results shown in Figure 19 shows that the pipe material near the magnetizer pole is saturated (permeability $\sim 15$ ), but the material midway between the poles is not (permeability $\sim 75$ ). Lower magnetization levels mean the tool is more sensitive to stress and material property variations. 


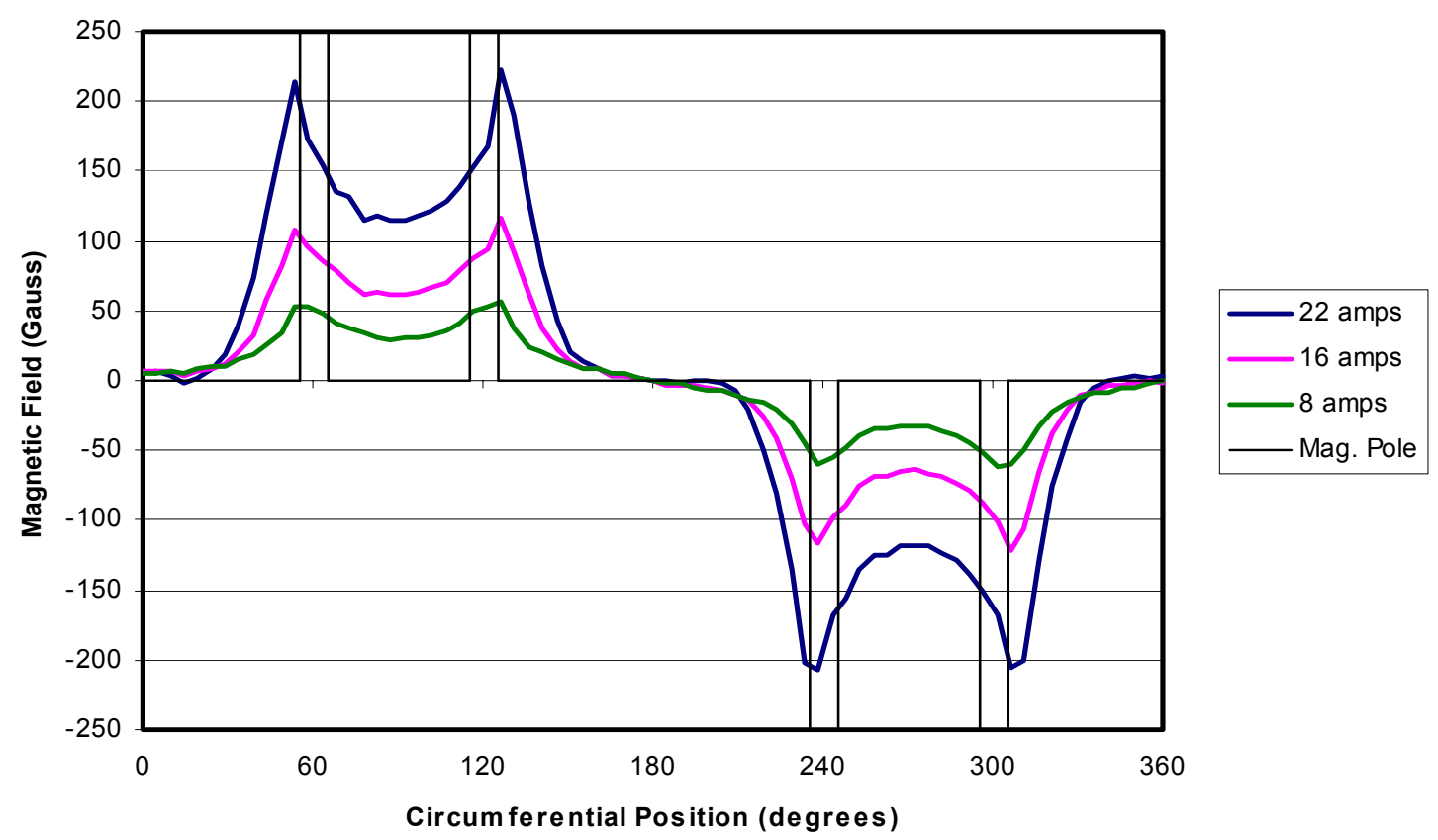

Figure 17. The radial component of the magnetic field around the circumference of the pipe for the electromagnet test bed vehicle circumferential magnetizer.

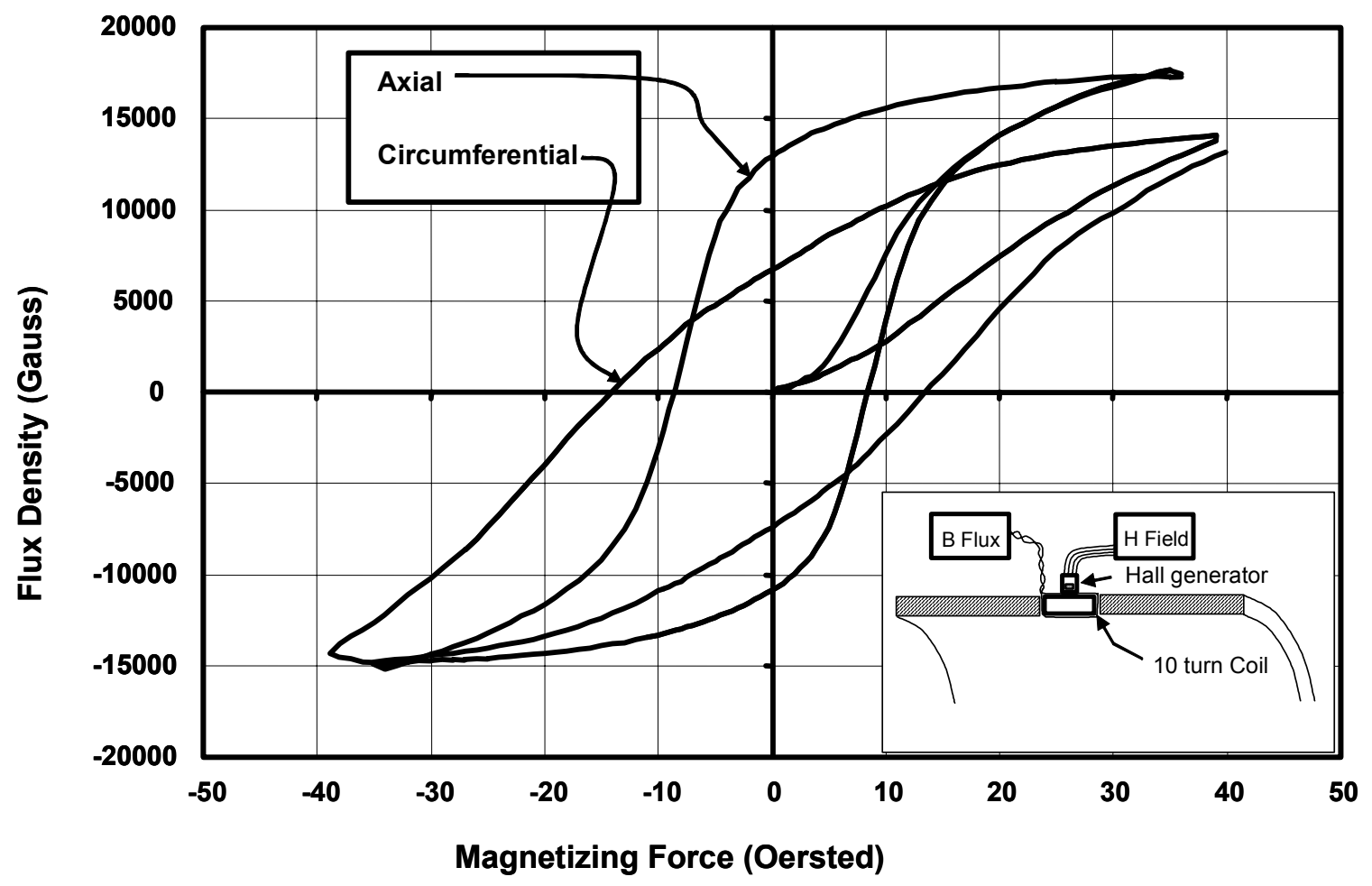

Figure 18. In-situ magnetic measurements demonstrating difficulty in saturating the pipe material in the circumferential direction. 
June 2003

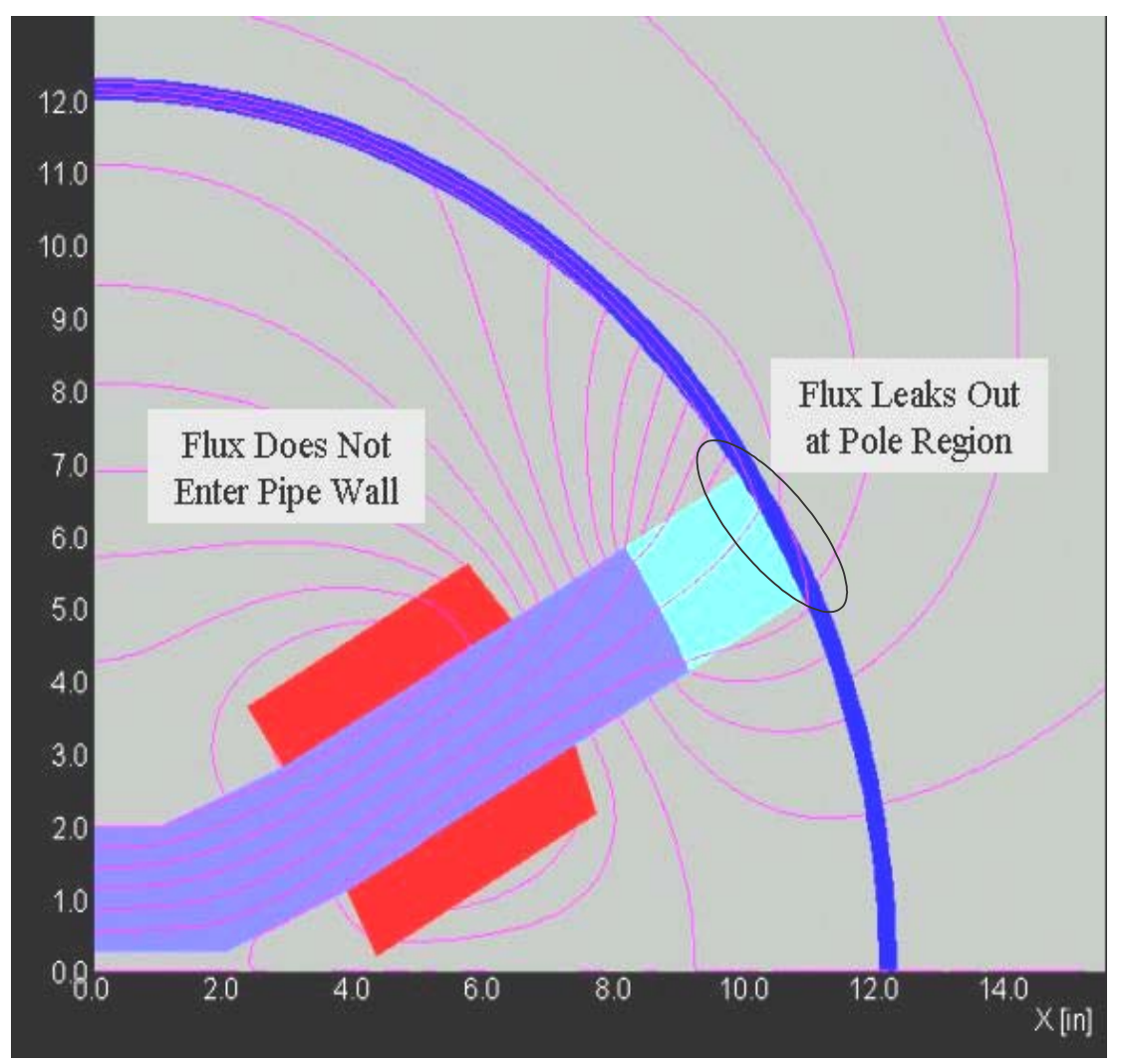

\section{Figure 19. Quarter magnetic finite element model electromagnet circumferential magnetizer.}

About 46 amps were required to attain 100 Oersted field levels in the pipe midway between the poles. A graph of field level versus current is shown in Figure 20. Initial modeling results suggested that only 17 amps would be required to attain 150 Oersted field levels. The discrepancy in modeling and experimental results appears to be the result of differences in magnetization properties in the circumferential and axial directions. The models used published magnetization curves $(\mathrm{BH})$ [6] for pipe material magnetized in the circumferential direction, but the curves were generated from coupons removed from the pipe, thus relieving many of the stresses associated with pipe forming process. The electromagnet will enabled magnetization in the circumferential direction and the ability to attain in situ magnetization curves for additional analyses. 
June 2003

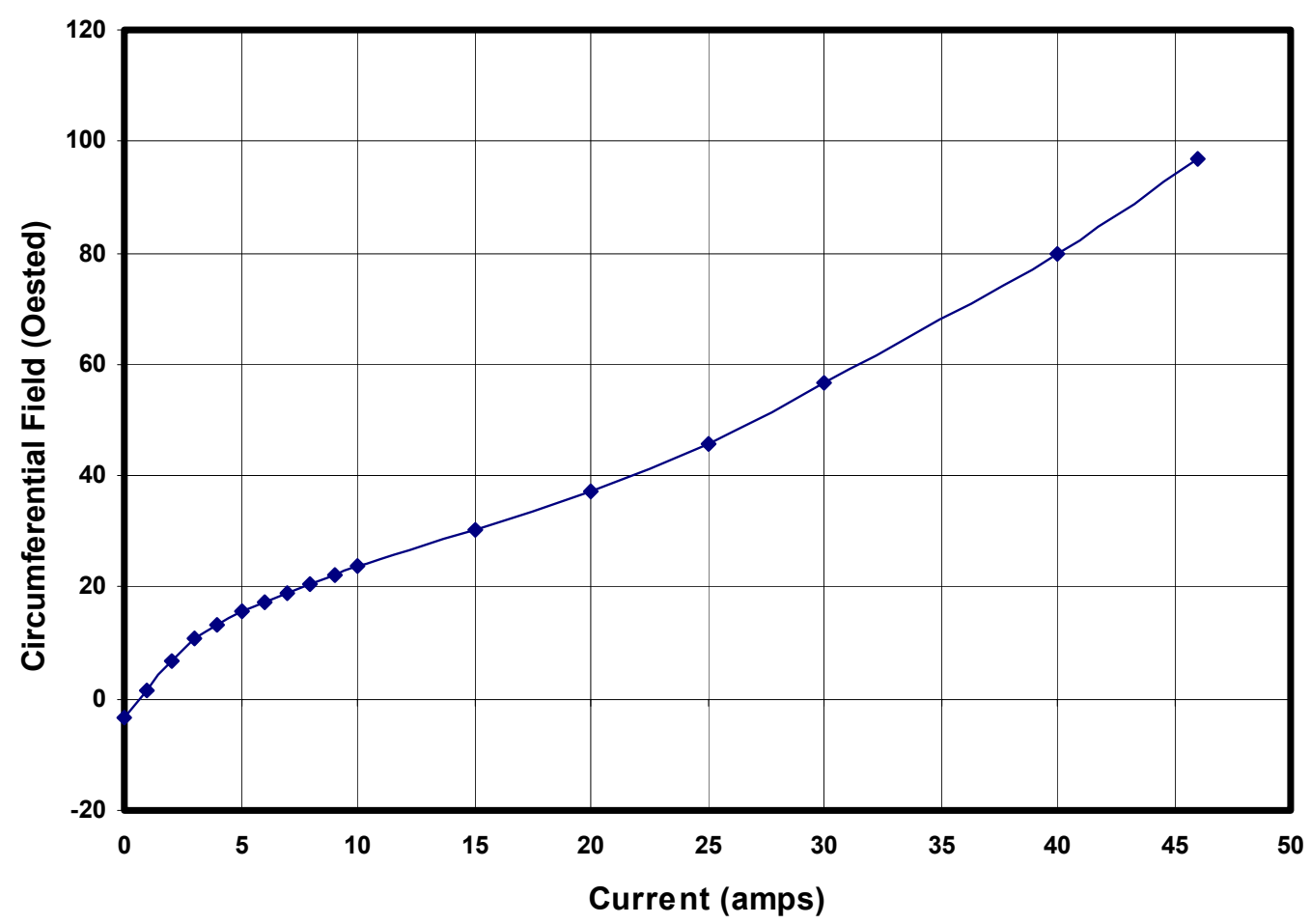

Figure 20. Field level in the LTR pipe segment as a function of current level.

Note: The initial negative level is the retentivity from prior magnetizations.

\section{Sensors}

Sensors between the magnet pole pieces measure the flux leakage field. The purpose of sensor systems is to convert the flux leakage field into a signal that can be stored and analyzed. The sensor system consists of the sensors themselves, the mounting system used to support the sensors, wear plates between the sensors and the pipe, and cabling between the sensors and data recording components.

Hall effect sensors are semiconductor devices that convert flux leakage signals into electrical signals. The sensors on the test bed vehicle were replaced by SS495 Series, solid-state, ratiometric, linear sensors manufactured by Honeywell Micro Switch. These sensors have the necessary characteristics to measure the fields generated by mechanical damage defects. The sensors operate on supply voltages ranging from 4.5 volts to 10.5 volts. Outputs are ratiometric, and are set by the supply voltage. The sensors measure a minimum of $+/-600$ Gauss, and they include an amplifier integrated into the circuit. Sensor arrays made up of multiple sensors are constructed using printed wiring boards. These sensor arrays are potted in a sensor housing and mounted in the sensor. Figure 21 is a photograph showing a sensor head with replaceable sensor circuit. More than one Hall effect sensor is contained in each sensor head assembly. Figure 22 shows a sensor head configured with four Hall effect sensors spaced evenly over the 1-inch wide sensor head. 


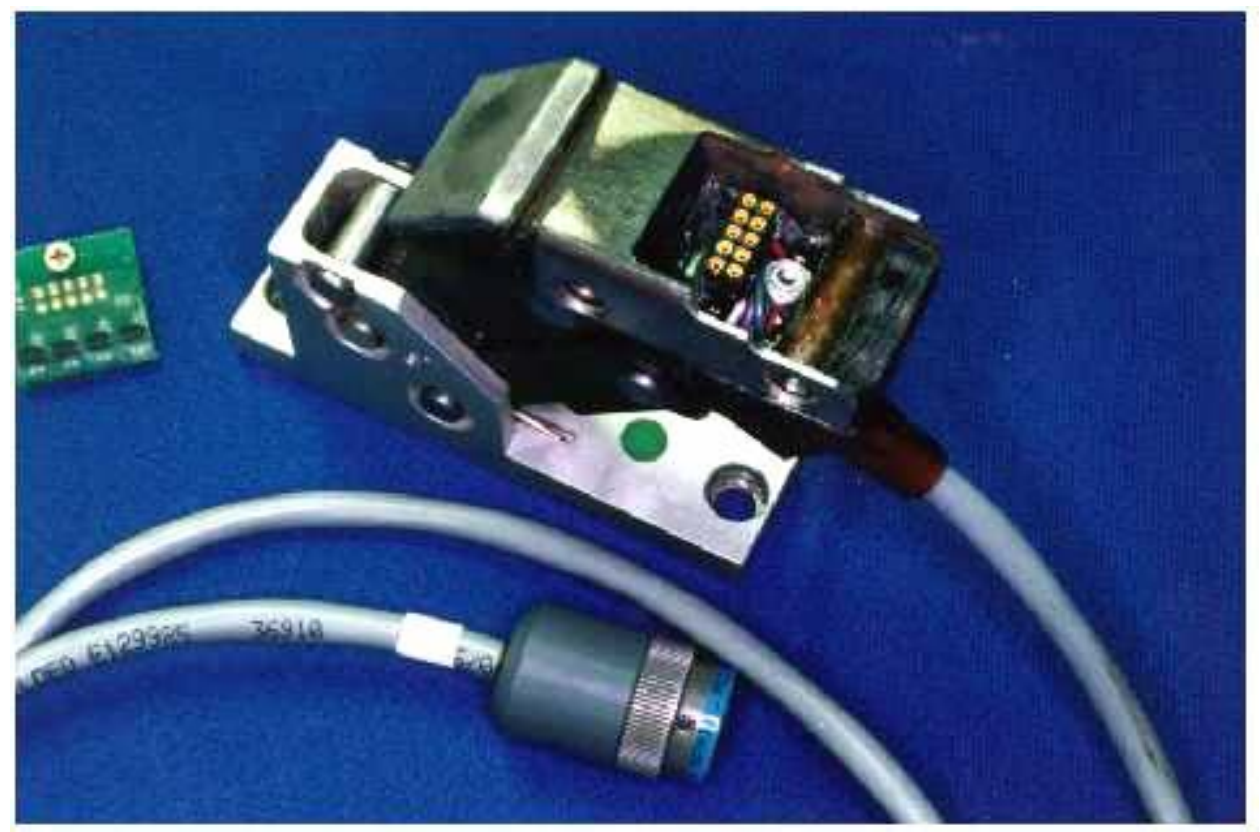

Figure 21. A sensor head with replaceable sensor circuit.

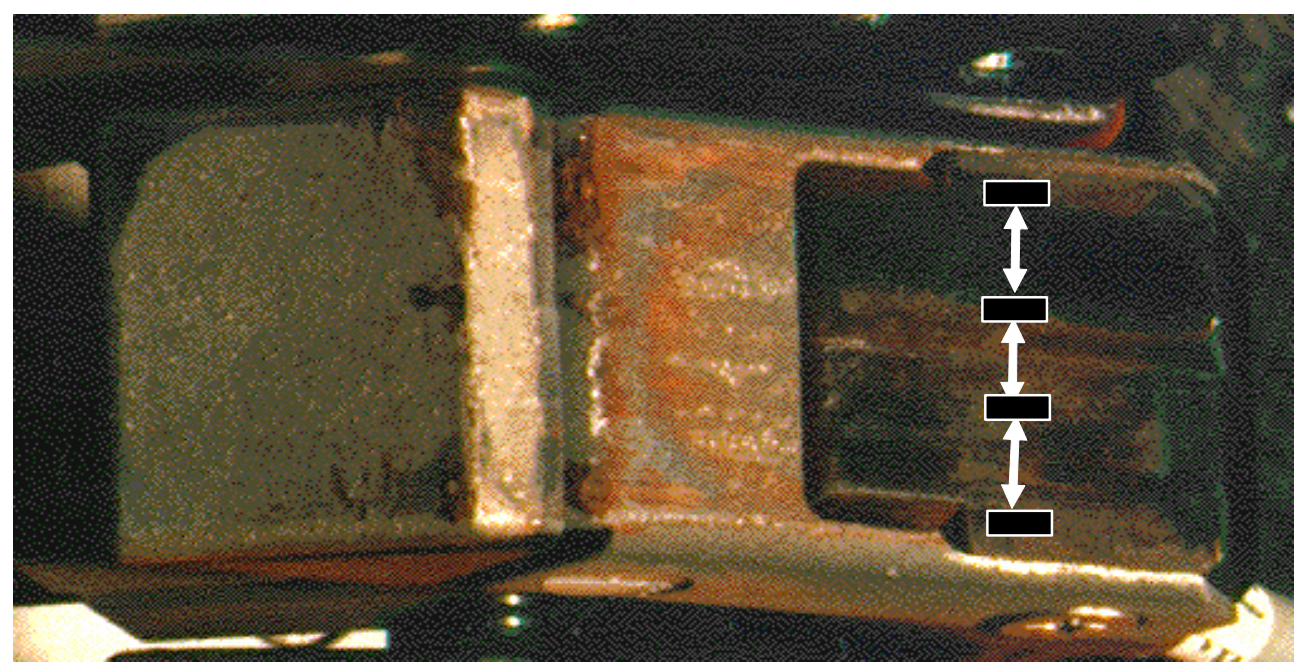

Figure 22. An early sensor system with four sensors per sensor head.

The sensor spacing has to be based on the flux leakage response of the defect. The sensor must reasonably capture the peak response of the defect. Figure 23 shows a flux leakage signal from a crack that measured single probe scanned in the circumferential direction across a series of cracks of different depths. The output of the sensor was sampled in 0.050 -inch intervals. The eight vertical lines that span the $70 \%$ deep crack show that eight sensors per inch does not quite adequately measure the peak. Detailed analysis showed that the peak was 84 gauss. The left sensor measured a peak of 78 gauss (a 7\% error) and the right sensor measure 74 gauss (a 12\% error). The other cracks showed that the width of the signal does not change significantly; however, the sharpness is reduced. 


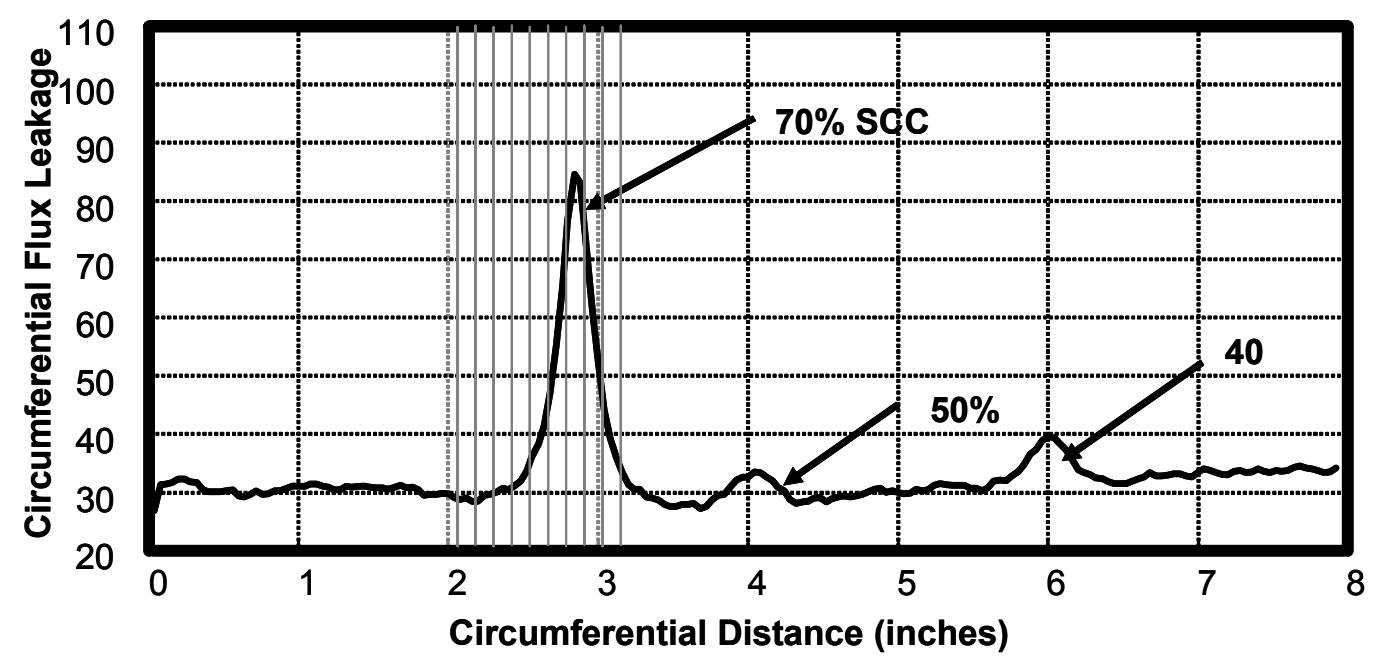

Figure 23. Sensor spacing determination based on crack signals acquired in the laboratory at 0.050 -inch circumferential intervals.

A sensor spacing of a sixteenth of an inch provides better sampling of the peak energy, with a maximum error of 2 percent observed. This sensor configuration is illustrated in Figure 24. More sensors are needed for circumferential MFL than axial MFL to achieve similar accuracies. For circumferential MFL, the flux spreads in the axial direction, which helps reduce axial sample interval but not sensor spacing requirements. Therefore to accurately record the flux leakage, the sensor spacing interval around the pipe is half that of axial MFL, typically between 2.5 to $5 \mathrm{~mm}$ ( 0.1 to $0.2 \mathrm{inch}$ ). For example, an axial tool with 250 sensors would have the same flux recording fidelity as a circumferential tool with 500 sensors. The sampling requirement along the pipe (in the axial direction) is helped by flux spreading, but most implementations stay with the same data interval as axial MFL to simplify data display, analysis, and overlay.

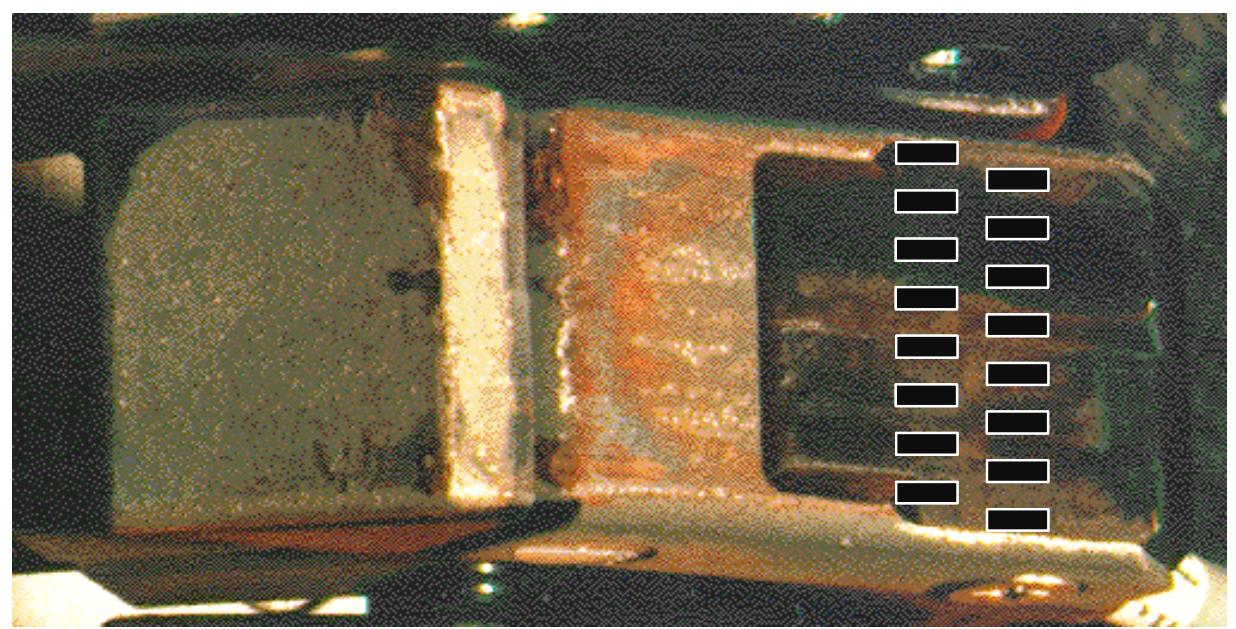

Figure 24. Dense sensor spacing for crack detection experiments with 16 sensors per one inch wide sensors head. 


\section{Data Recording}

The electronic components were personal computer compatible components using PC-104 format cards to minimize size and maximize ruggedness. The computer module shown in Figure 25 has a central processor unit (CPU) card, with six analog-to-digital (A/D) cards, and a power supply module. A mass storage device and cabling complete the data acquisition system.

The CPU module controls the functions of the data acquisition system. Sensor voltages are in the range of 0 to 10 volts. Using a 16-bit converter allows 65536 discrete voltage levels to be measured, equating to $0.15 \mathrm{mV}$ per bit. The respective A/D channel captures the data from 96 sensors, the $96 \mathrm{~A} / \mathrm{D}$ channels being achieved by using six A/D cards, each with 16 input channels. Coupled to the CPU is a solid-state disk drive storage device that stores the control software and the acquired data. This solid-state storage device uses Flash memory as the storage elements, and includes a standard IDE interface. Using non-volatile memory configured to have the standard disk interface results in a rugged storage device with no development of custom hardware.
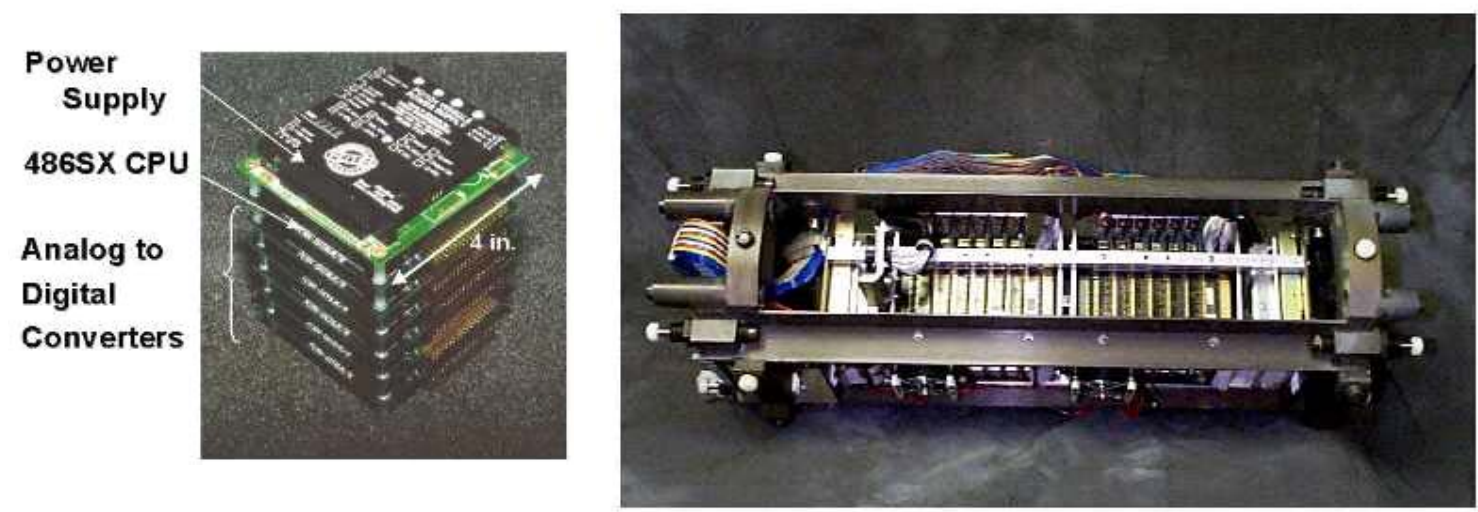

Figure 25. The data recording system (left) is mounted in a shock and vibration damping cage (right).

The digital sensitivity of the sensor is 0.1 gauss per quantum level. The system noise is one or two bits or 0.4 gauss. In previous experiments, the random signals due to pipe roughness, material variation and other sources was typically 1 or 4 gauss. Therefore, the recording system has sufficient fidelity to record the smallest meaningful signal.

\section{Data Display}

A data display convention was established for visualizing the flux leakage from defects. The convention, illustrated in Figure 26, is used in many of the figures in this report and is one of many used by commercial pipeline inspection companies. In this representation, the inspection tool moves from left to right. The sample interval on the horizontal axis is $0.1 \mathrm{inch}$. On the vertical axis, the sensor spacing is 0.0625 inches (a sixteenth of an inch). Color is used to indicate flux leakage intensity. White is assigned to be the nominal background magnetization level. Increasing flux leakage levels progress through green, yellow, orange, red and black. Flux levels below the magnetization level are represented by shades of blue. 
June 2003

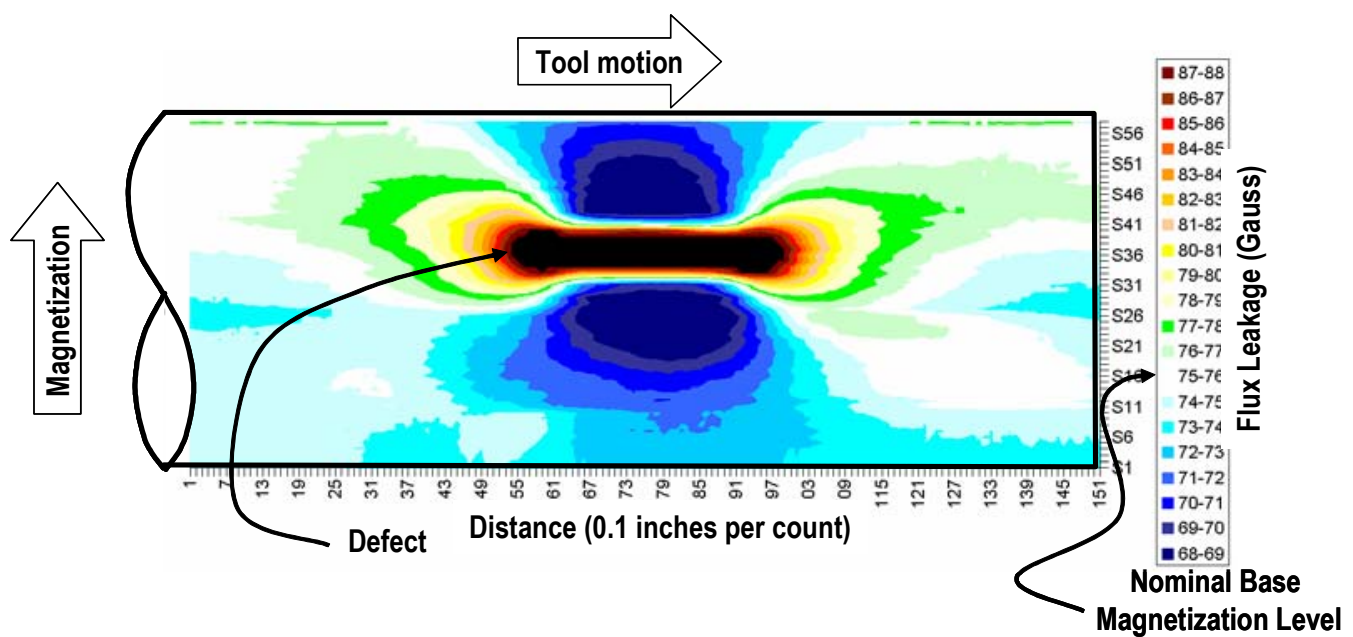

Figure 26. Data display convention. 


\section{RESULTS}

Two technology enhancements to circumferential MFL technology were tested to determine their utility in improving detection of cracks. The methods were:

- Combining high- and low-magnetization technology for stress detection

- Combining axial and circumferential MFL.

Combining high- and low-magnetization technology, as is being done for mechanical damage, was examined to determine of stress patterns around defects could be detected; these stress patterns may contain critical information on the severity of cracks and crack-like defects. Combining axial and circumferential MFL was examined to evaluate improvements in crack detection and distinguishing cracks for axially oriented volumetric defects.

\section{High ANd Low Magnetization Technology}

Combining high and low magnetization technology was tested using EDM notches and weld deposition cracks under stresses near yield around the notch/crack tips. These were conducted at:

- Pressures of atmospheric, 250 psi and 500 psi

- Magnetization levels of 75 Oersted and 40 Oersted

Prior experiments showed that magnetization levels above 120 Oersted are required to eliminate stress effects from the flux leakage signal. However, 75 Oersted was the highest level that could be attained in this pipe material. While analysis showed that these field levels were not sufficient to apply the previously developed high- and low-magnetization processing schemes, the tests were conducted to examine what other effects could be detected and evaluated.

\section{Detection of Notches}

The detection of notches in the parent material and weld zones demonstrates the sensitivity of the circumferential MFL system as configured. Figure 27 shows a typical result from the pipe sample with EDM notches. Two large notches and four smaller notches were detected. There is also a large zone between the two circled regains that have no defects. This area can be used to establish a noise level for the detection of smaller defects. Figure 28 focuses on the right portion of Figure 27. The 2-inch long, 50 percent deep EDM defect was clearly detectable. To the right of this defect is a 2-inch long, 10 percent deep EDM notch that produces a signal that is three gauss above the magnetization level. In the left portion of this image were signals from two parallel notches that were 4 inches long and 10 percent that were overlapped for 2 inches. The image shows two signals at separate circumferential locations, but these signals merge at the overlap. 
June 2003

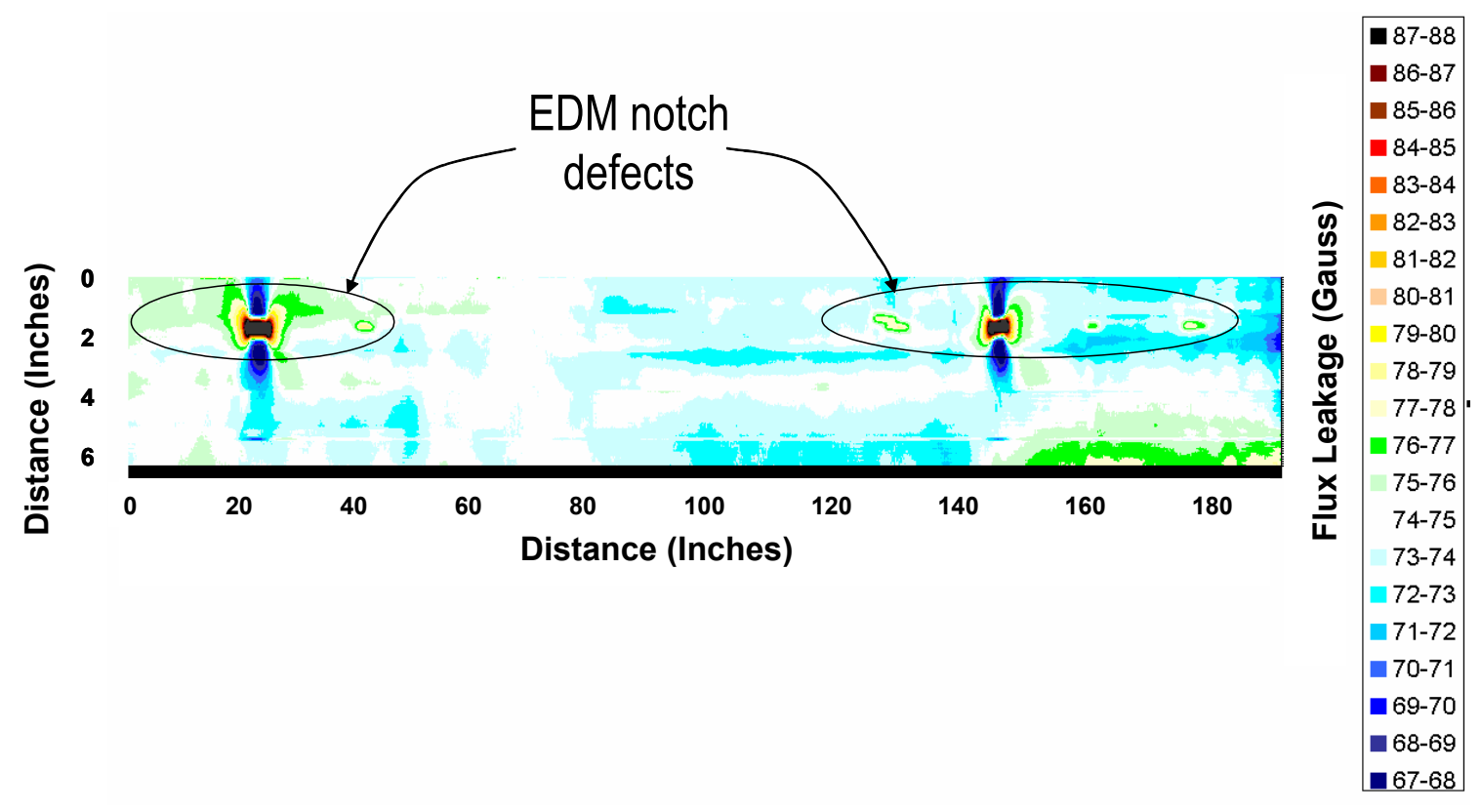

Figure 27. Flux leakage for the full pipe sample with EDM notches.

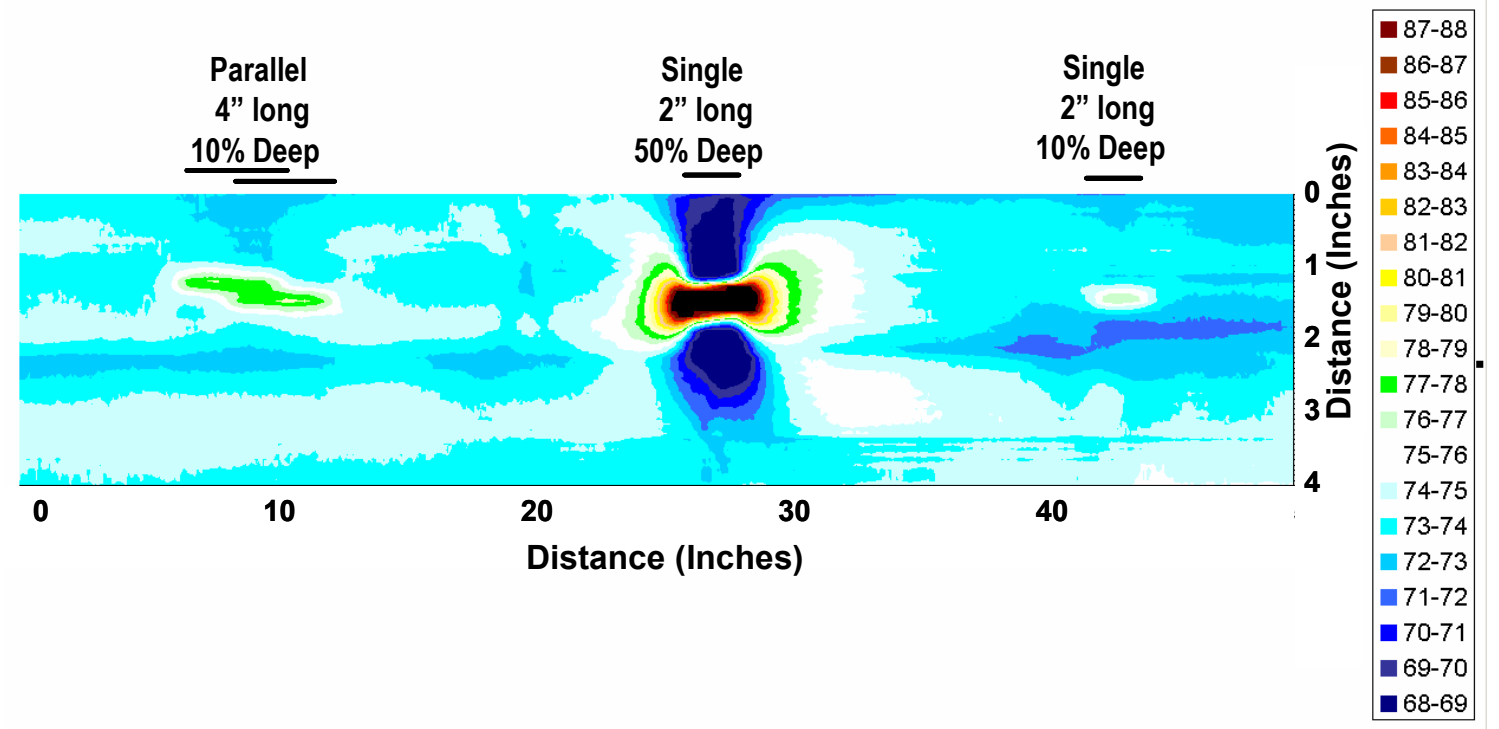

Figure 28. Flux leakage from three EDM notches demonstrating the detectability of these defects. 
EDM notches were detected in the weld zone. However, the detection process is complicated by signals from the weld itself. Defects in the weld must produce a signal that is distinguishable from the ever present weld signal. To extract the defect signal, a typical weld signal can be generated by averaging recorded data from several axial measurement positions. The number of averages depends on situation, but can be as small as four axial readings, which corresponds to a length of about $10 \mathrm{~mm}(0.4 \mathrm{inch})$ in many circumferential MFL tools. More commonly, over 100 axial readings are used, which corresponds to $250 \mathrm{~mm}$ (10 inches), or measurements from the entire pipe seam of a joint of pipe are used. To detect defects, the average weld signal is subtracted from the measured signals. The difference shows both the natural variations in the welding process and potential defects. Since seam welds are made with automatic processes, the natural variations are small in amplitude and long in axial extent as compared to cracks and corrosion in this zone. Figure 29 shows a typical result from the weld zone with four EDM notches and one slag inclusion. The three larger defects can be seen. However, the 10 percent defect EDM notch was not distinguishable within the weld signal. One complicating factors is inspection tools often rotate as the travel down a pipeline. The generation of an average and subsequent subtraction is valid over a short range. Figure 29 shows that the weld commensuration procedure works only over a short range. The blue and red stripes before and after the defect zone are caused by tool rotation.

\section{Seam weld notches detectable Tool rotation causes striping}

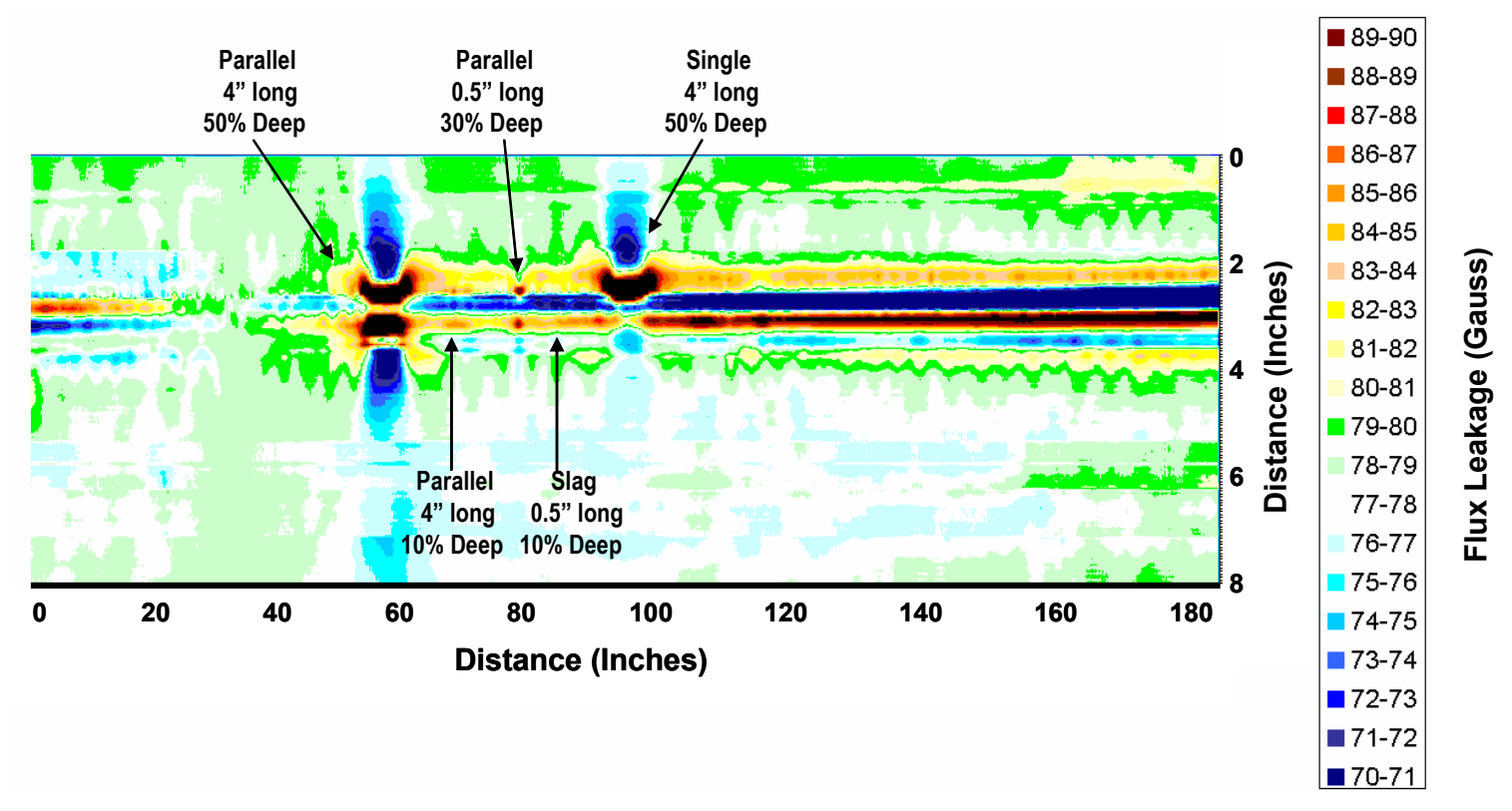

Figure 29. Flux leakage from a weld zone with EDM notches.

\section{Stress Effects}

The processing of high- and low-magnetization signals assumes there would be a difference in signal strength at these magnetization levels. Prior results using circumferential MFL to examine cracks showed an increase in signal was produced at stress levels approaching yield. This 
increase in signal could be due to stress effects or a stronger magnetic dipole caused by an increased crack opening. While sufficiently high field levels could not be attained to perform this type of analysis, it was still possible to assess if signal change were due to stress. For cracks under pressure, mechanical finite element modeling showed the surfaces separate an insignificant amount for notches. However, for tight cracks, any additional opening would increase signal strength.

The flux leakage from notches at the three pressure levels was analyzed to determine whether stress effects would help detection of cracks. Stress should affect flux leakage signals all attainable magnetization levels since these are below saturation. Figure 30 shows a typical result. All cracks analyzed show a similar result, that signal amplitude decreases for increasing internal pressure. The stress slightly decreases signal amplitude. Prior results on natural SCC show that stress increased signal strength. Combining these results indicated that:

- The increase in signal from the natural cracks is due to increased opening

- Any stress effect will diminish the crack signal.

Therefore, the stress induced changes in flux leakage do not improve detection of SCC. Furthermore, while stress effects can be observed, multiple magnetization methods to detect cracks will not be useful because the effects are small compared to variable magnetization level associated with circumferential MFL. To increase signal strength, in-line inspections should occur at maximum operating pressure to open cracks and as high a magnetization level as possible to reduce to effect of stress.

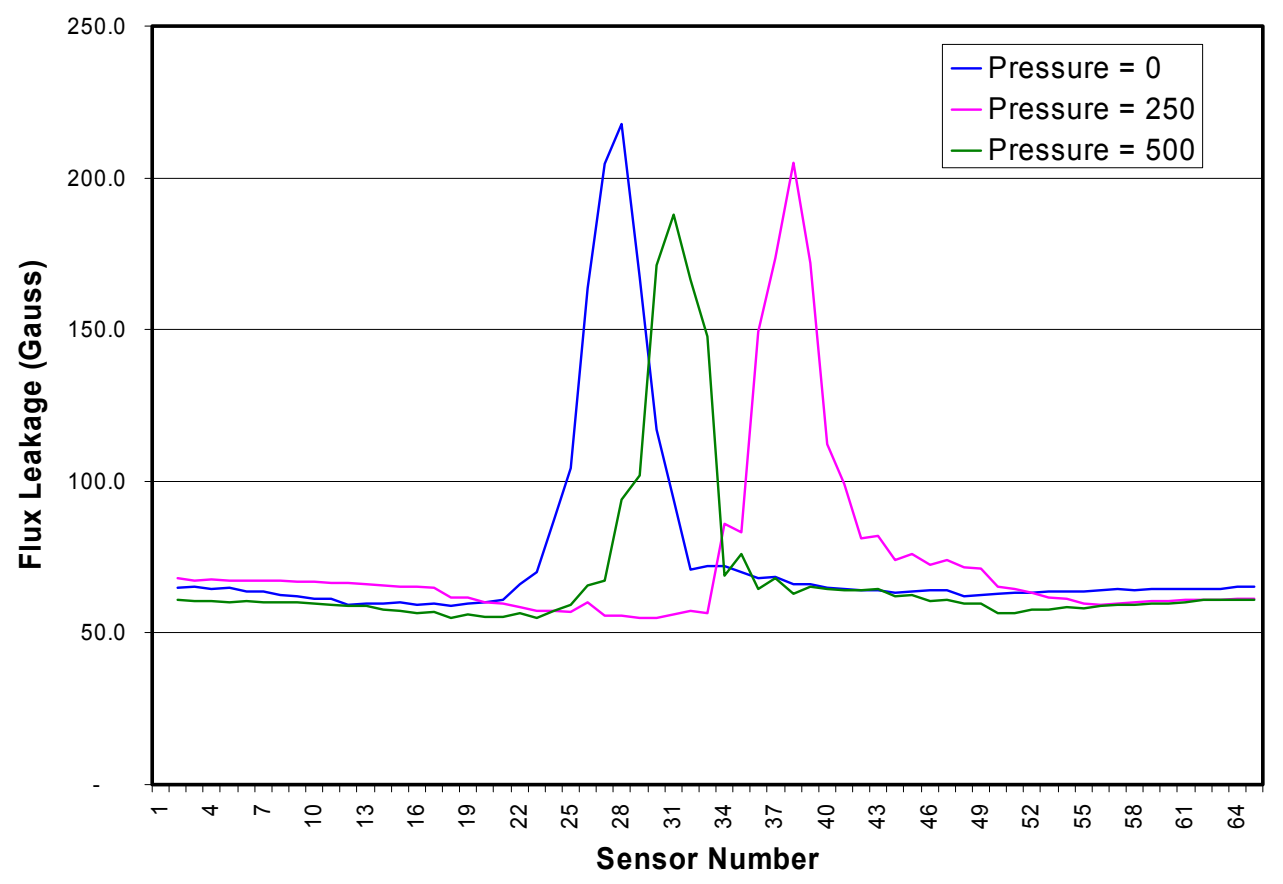

Figure 30. Flux leakage signals from a fifty percent deep EDM notch at pressures ranging from atmospheric to approaching yield. 


\section{Weld Deposition Cracks}

Inspection technology developers often have difficulty obtaining natural samples for testing, so they turn to artificial defects. The most common representation of cracks, EDM notches, produces a much stronger flux leakage signals than natural SCC. This extra flux leakage is due to the width of the notch as compared to the negligible opening of a natural crack. Weld deposition cracks are as tight as natural stress corrosion cracks. However, the installation process can affect the magnetic properties.

Weld deposition cracks were available in the pipe sample and flux leakage signals were recorded from these defects. Figure 31 shows the results from one row of cracks. The signals are much more volumetric than the notch signals and appear to have very similar amplitudes. Table 1 shows the depth of the cracks. The depths of these cracks were measured using two ultrasonic methods, time of flight diffraction (TOFD) and phased array imaging. Neither technique could accurately size the depth for the second crack. These results show that weld deposition cracks are not good substitutes for natural cracks for magnetic inspection systems.
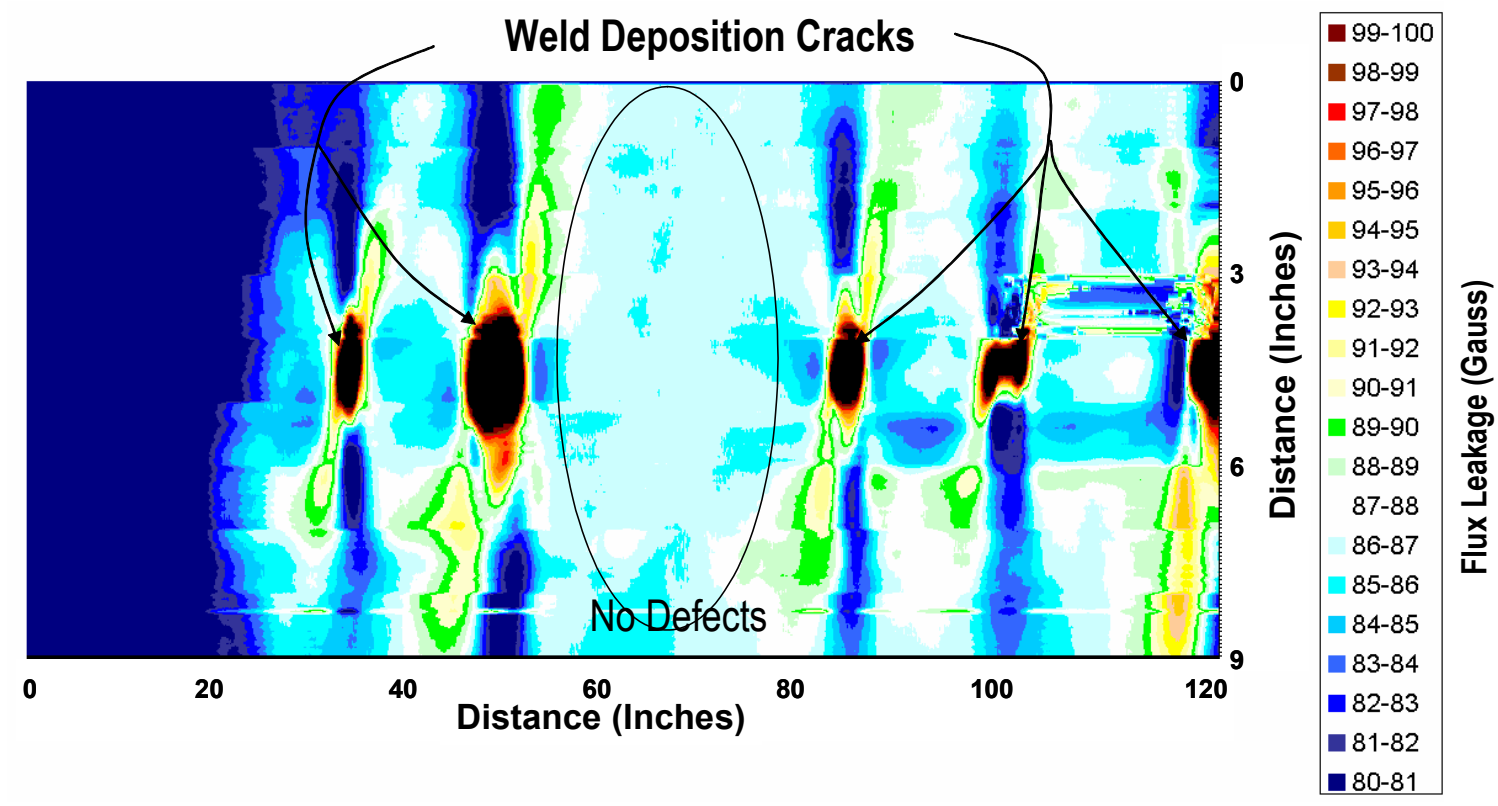

Figure 31. Flux leakage from weld deposition cracks.

Table 1. Crack Depths

\begin{tabular}{|c|c|c|c|c|}
\hline Crack \# & ID & Target & UT TOFD & UT Phased \\
\hline 1 & $\mathrm{~A}$ & $10 \%$ & $23 \%$ & $8 \%$ \\
\hline 2 & $\mathrm{C}$ & $10 \%$ & & \\
\hline 3 & $\mathrm{G}$ & $30 \%$ & $32 \%$ & $19 \%$ \\
\hline 4 & $\mathrm{I}$ & $70 \%$ & $70 \%$ & $71 \%$ \\
\hline 5 & $\mathrm{~K}$ & $50 \%$ & $32 \%$ & $38 \%$ \\
\hline
\end{tabular}




\section{Natural Stress Corrosion Cracks}

Prior results showed that some natural cracks could be detected with circumferential MFL. These were laboratory results under static conditions. A pipe sample with natural SCC was examined using the circumferential inspection system. Figure 32 shows the flux leakage from the pipe sample, a magnetic particle enhanced image of the crack zone and photograph indicating corrosion pits. Figure 33 shows the details of the crack zone. It should be noted that not all cracks were detected and the depth of these cracks was not known. The pipe sample had numerous corrosion pits and multiple cracks that look like corrosion in the flux leakage image.

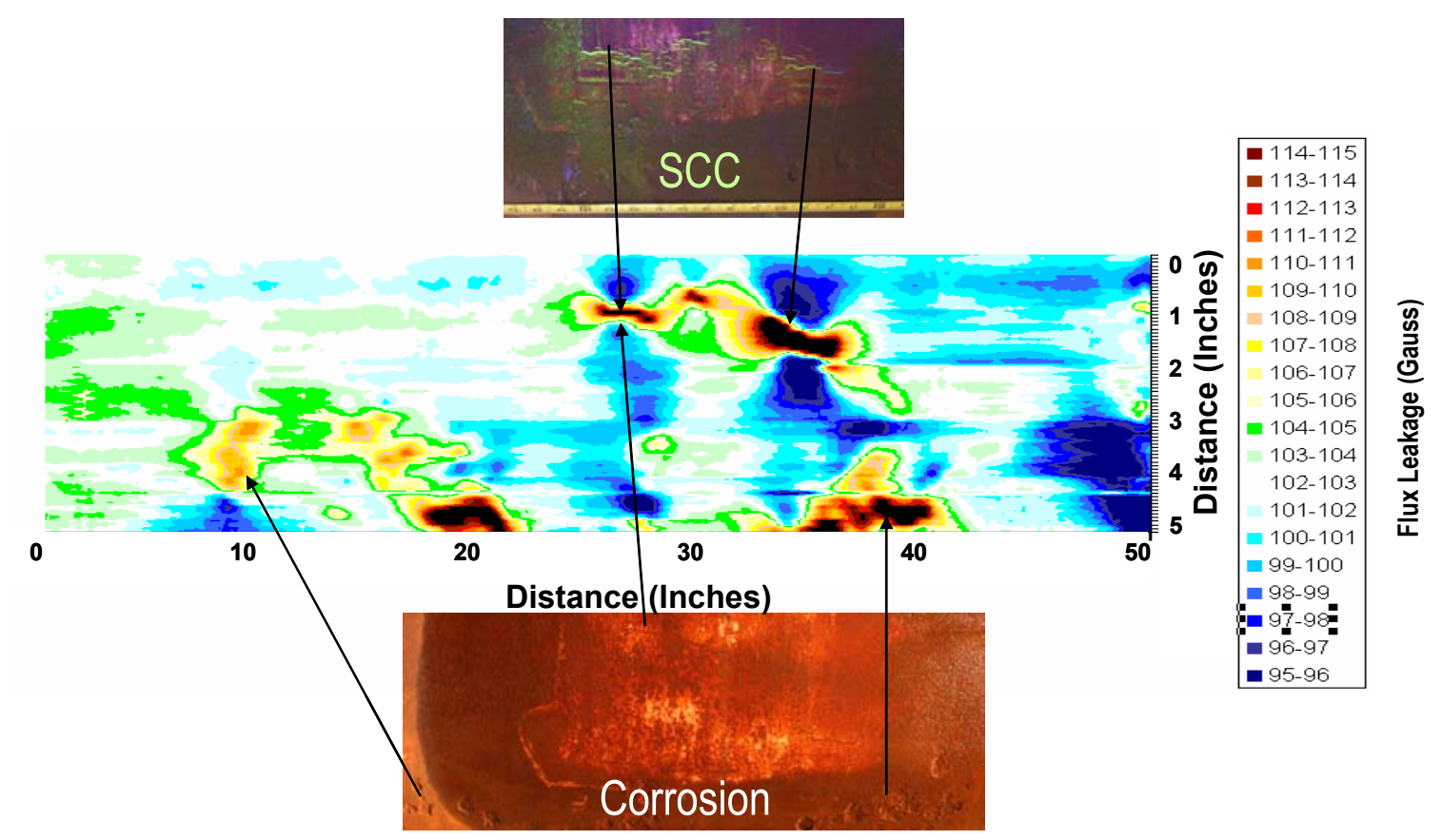

Figure 32. A flux leakage image, magnetic particle enhanced photograph of a stress corrosion crack colony and a photograph indicating corrosion pits. 


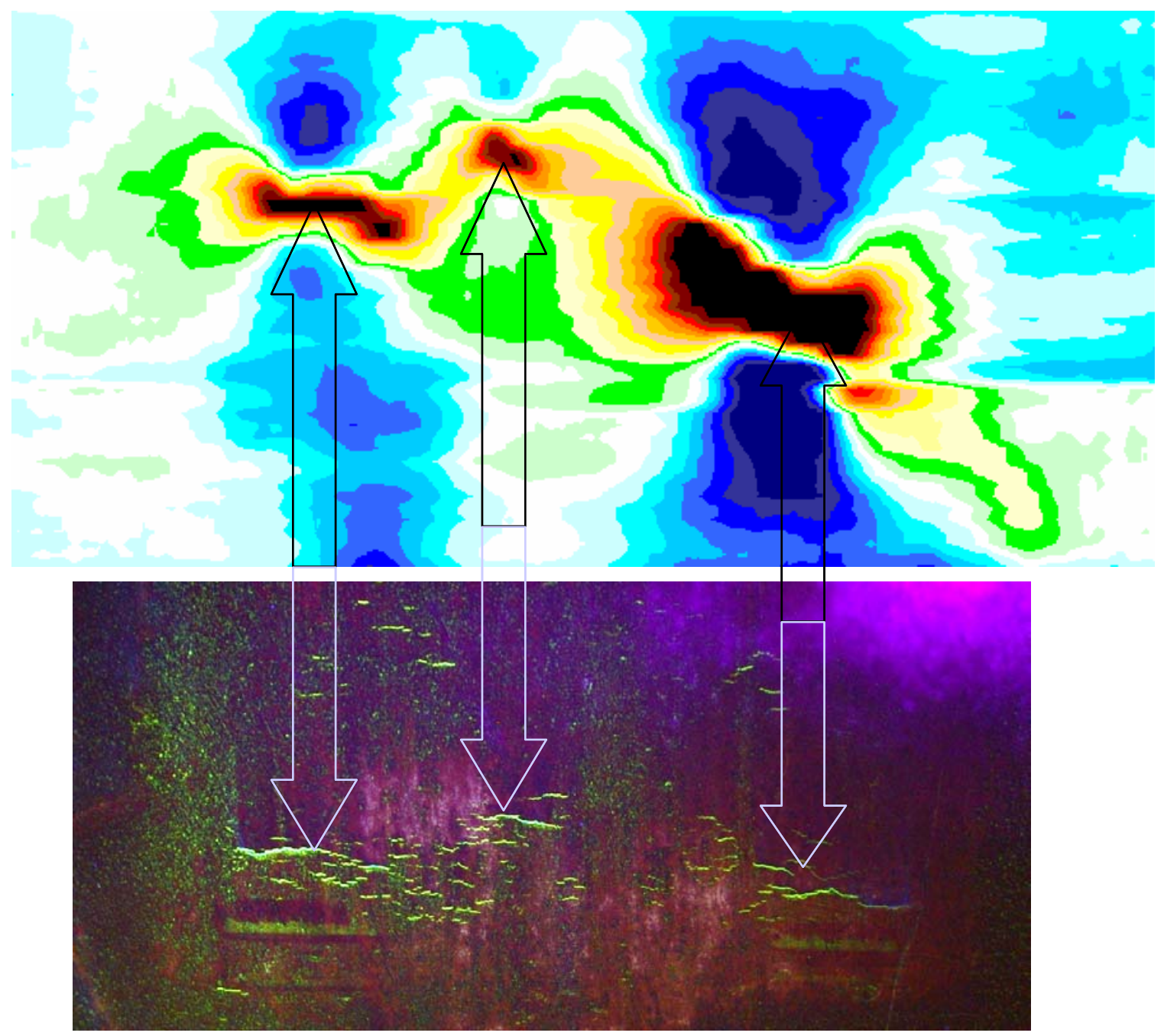

Figure 33. Close-up of a flux leakage image and magnetic particle enhanced photograph of a stress corrosion crack colony.

\section{Combining Circumferential AND AXIAL MFL}

Combining data from two inspection technologies often provides improved inspection results. The natural SCC sample was examined with the axial MFL inspection vehicle that is part of the PSRC. A comparison of flux leakage difference from cracks and pits, and noise signal differences are provided in the following sections.

\section{Noise Differences}

Detection of cracks depends on both the strength of signal from the smallest significant defect and the background noise of the material. The single channel data presented in Figure 34 shows both the noise differences and the repeatability of multiple pulls. For the axial data in the lower portion of the figure, strong repeatable signals can be seen between the 3 metal loss defects of 5 , 
10 , and 20 percent. These signals are a result of variations in magnetic properties and wall thickness in a pipe. The noise levels are approximately 3 gauss. This signal level is similar to that from a 1-inch diameter 5 percent deep pit, but it is an order of magnitude less than that from a 1-inch diameter 20 percent deep pit.

The data from the circumferential tools shows the same pipe sample has much lower noise signals. The noise levels are less than a gauss. Again, the pull results are very repetitive. The images presented in Figure 35 show this could improve defect detection. A few gauss signal may get detected in the circumferential MFL image, but may escape detection in the axial image. This lower relative noise level will aid circumferential MFL in the detection of small defects.

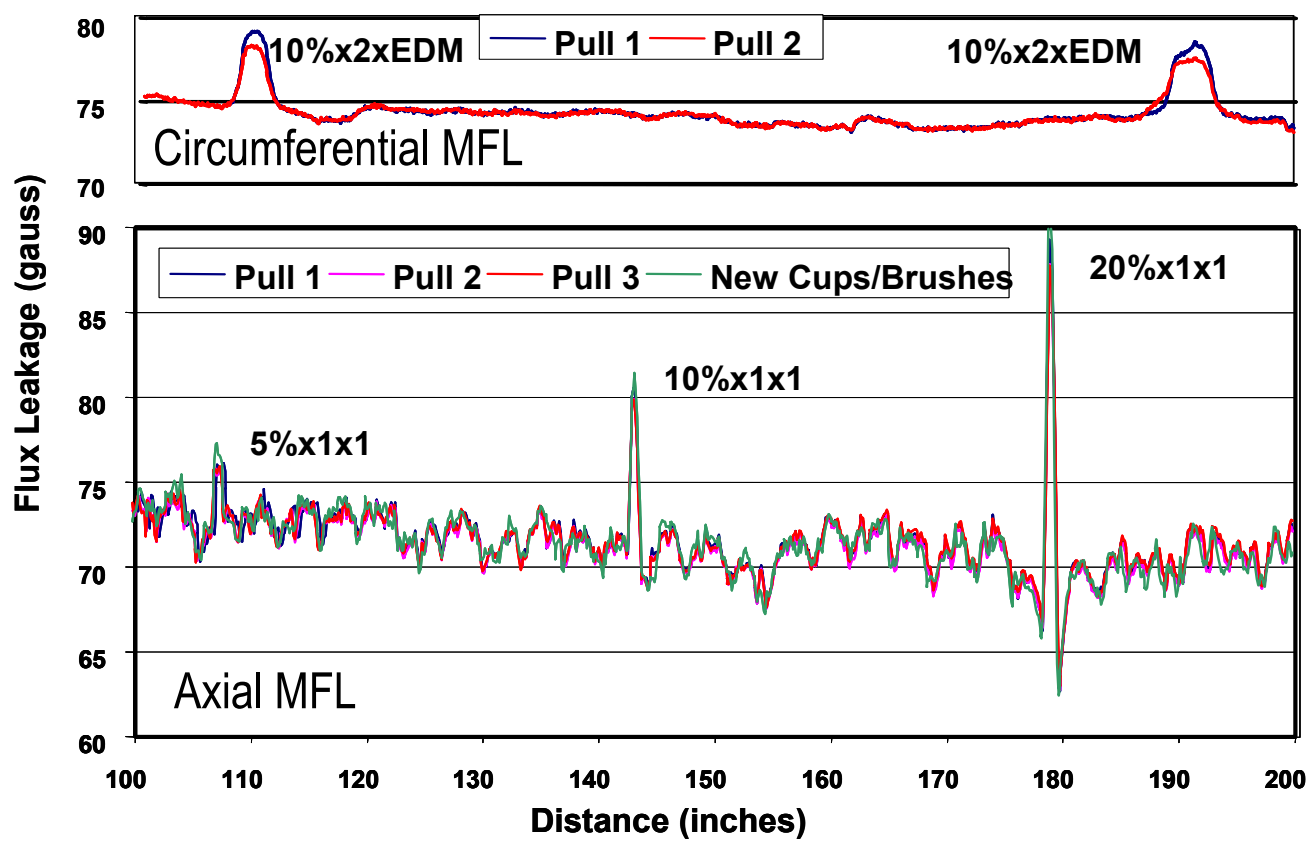

Figure 34. Single channel axial and circumferential flux leakage data showing both the noise differences and the repeatability of multiple pulls. 


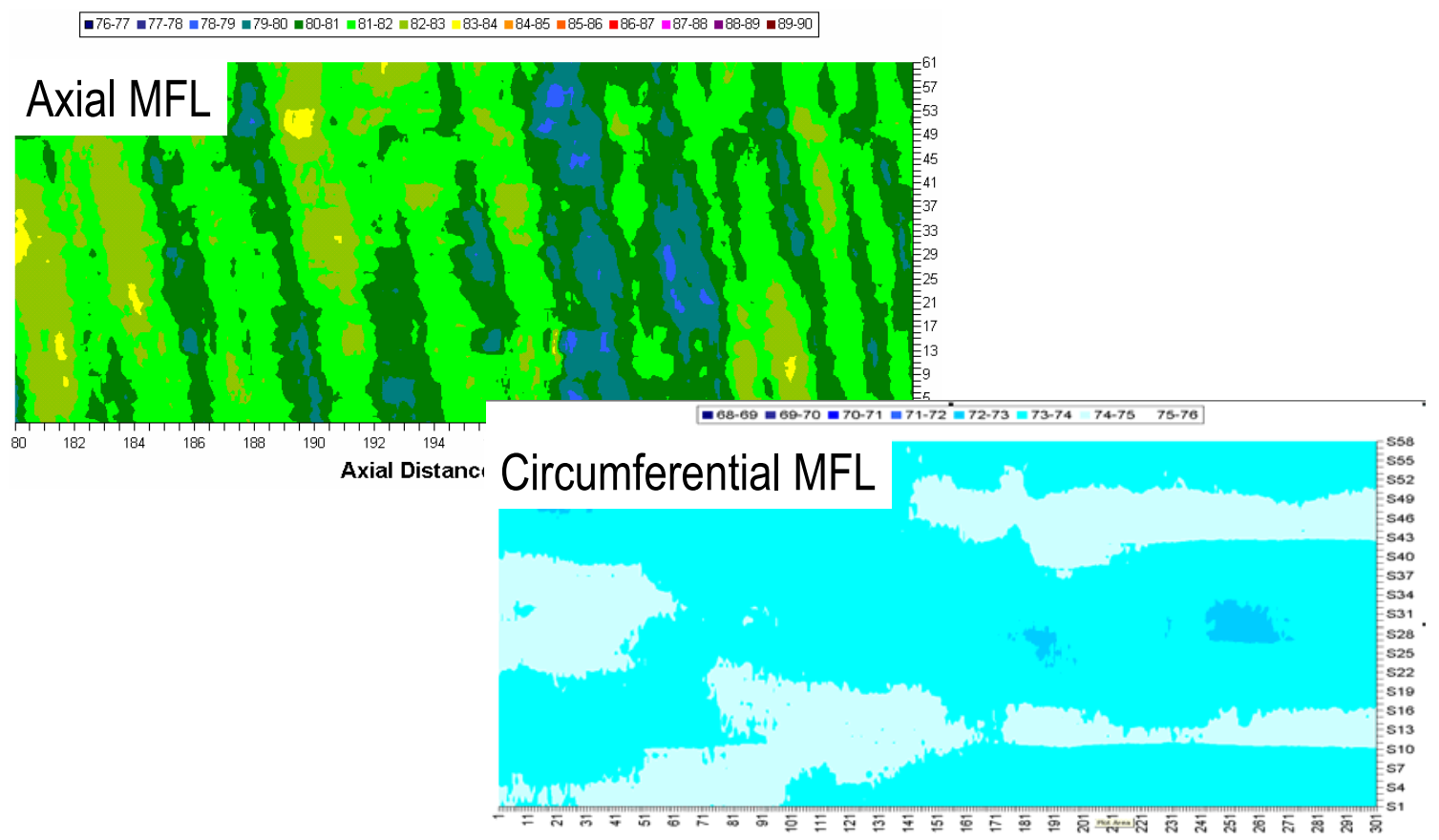

Figure 35. Axial and circumferential flux leakage image showing both the noise differences.

\section{Flux Leakage Differences}

A pipe with natural SCC and corrosion pits was examined with both circumferential MFL and the more traditional axial MFL. Figure 36 shows the flux leakage data for both inspection methods. The pipe sample had both stress corrosion cracks and numerous corrosion pits. The corrosion pits, concentrated between the 8:00 and 8:30 clock positions (in Blocks 1-8), were detected with both implementations of MFL technology. Note that the two inspection methods produce different images of the corrosion pits since the pits are aligned along the axis of the pipe. For axial MFL, the pits appear as a series of distinct defects less than an inch long. For circumferential MFL, the flux leakage from neighboring pits merges, causing the defects to appear as one longer axial defect. While the flux leakage maps for both methods show the detection of pits, only the circumferential MFL could detect some of the crack colonies, which were between the 8:30 and 9:00 position (in Blocks A, B and C). Also, note that for circumferential MFL, multiple cracks look like corrosion. However, the lack of axial signals in the regions of the cracks illustrates that a combination of axial and circumferential signals can be used to detect and identify some cracks. It should be noted that the method did not detect all cracks and the depth of these cracks was not known. The cracks may have be opened and deepened when pipe reduced to 24 inches in diameter from 26 inches in diameter. 
June 2003
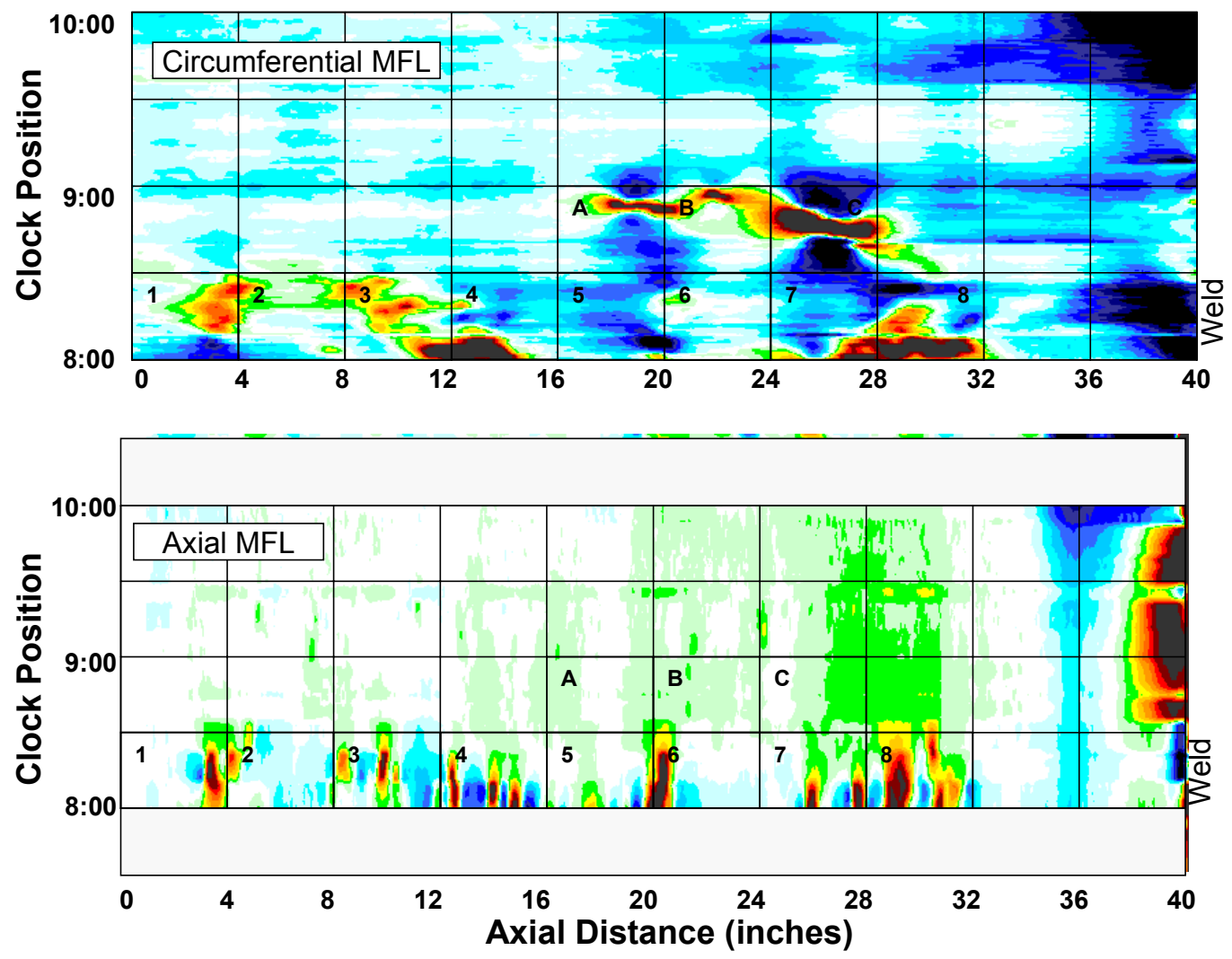

Figure 36. Circumferential and axial flux leakage image of stress corrosion crack colonies and corrosion pits. 


\section{CONCLUSIONS}

To overcome deficiencies of the more conventional axial MFL, circumferential MFL technology changes the orientation of the magnetizing field to improve detection and sizing of axially aligned defects. However, this simple solution has implementation difficulties that reduce the performance capability. Even with the field aligned properly, this technology has limitations detecting cracks on the outside surface that have the potential to grow to failure. Two technology enhancements to circumferential MFL technology for improving detection of cracks were tested. The first approach to improve circumferential MFL technology examined combining high- and low-magnetization technology to detect stress changes caused by cracks. While this method has shown promise for characterizing gouges cause by third party excavation equipment, it was unsuccessful for improving crack detection for two reasons. First, the stress diminishes the crack signal, while the opening of the crack increases the signal. The stressinduced changes in flux leakage around cracks were weak and any critical information on the severity of cracks and crack-like defects is difficult to distinguish from changes caused by the crack opening and other inspection variables. Second, it is difficult to magnetize pipe material in the circumferential direction. A relatively low, non-uniform magnetization level produced by the circumferential magnetizer makes detection of changes due to stress extremely difficult. The second approach to improve circumferential MFL technology examined combining axial and circumferential MFL to improve crack detection and distinguishing cracks for axially oriented volumetric defects. While successful results were presented, circumferential MFL can only detect larger cracks.

Circumferential MFL can be used to detect many corrosion, mechanical damage, and crack defects. However, the detection capabilities and sizing accuracies may not be sufficient for all pipeline threats. Inspection tools that use more sophisticated technologies for detecting and sizing defects may have better performance capabilities, but will likely be expensive to operate. Circumferential MFL will be useful in identifying locations for detailed testing. While performance enhancements may be limited, circumferential MFL inspections will be part of the inspection process for many decades. 


\section{REFERENCES}

1. Bubenik, T. A., Stephens, D. R., Leis, B. N., and Eiber, R. J., Stress Corrosion Cracks in Pipelines: Characteristics and Detection Considerations, Battelle, Report Number GRI95/0007 to the Gas Research Institute, April 1995.

2. Nestleroth, J. B. and Bubenik, T. A., Magnetic Flux Leakage (MFL) Technology for Natural Gas Pipeline Inspection, Battelle, Report Number GRI-00/0180 to the Gas Research Institute, February 1999.

3. Dobmann, G. and Höller, P., "Physical Analysis Methods of Magnetic Flux Leakage," in Research Techniques in Nondestructive Testing, Volume IV, edited by R. S. Sharpe, Academic Press, 1980.

4. Atherton, D. L., et al, "Stress Induced Magnetization Changes of Steel Pipes-Laboratory Tests," IEEE Transactions on Magnetics, Volume MAG-19, Number 4, July 1983.

5. Atherton, D. L., "Effect of Line Pressure on the Performance of Magnetic Inspection Tools," Oil \& Gas Journal, October 27, 1986.

6. Nestleroth, J. B., and Crouch, A. E., Variation of Magnetic Properties in Pipeline Steels, Report No. DTRS56-96-C-0010, Subtask 1.1 Report, to the U. S. Department of Transportation, March 1998

7. Bubenik, T. A., et al, In-Line Inspection Technologies for Mechanical Damage and SCC in Pipelines - Final Report, Department of Transportation Report DTRS56-96-C-0010, June 2000.

8. Crouch, A. E., "Using MFL Corrosion Signals to Measure Pipe Wall Stress," The Pipeline Pigging and Repair Conference, Clarion Technical Conferences, Feb 2003.

9. Nestleroth, J.B., Evaluation of Circumferential Magnetic Flux for In-Line Detection of Stress Corrosion Cracks and Selective Seam Weld Corrosion, PRCI Report L51811, 1999. 